\title{
Nonlinear rheometry of entangled polymeric rings and ring-linear blends
}

Parisi, Daniele; Kaliva, Maria; Costanzo, Salvatore; Huang, Qian; Lutz, Pierre J.; Ahn, Junyoung; Chang, Taihyun; Rubinstein, Michael; Vlassopoulos, Dimitris

Published in:

Journal of Rheology

Link to article, DOI:

10.1122/8.0000186

Publication date:

2021

Document Version

Peer reviewed version

Link back to DTU Orbit

Citation (APA):

Parisi, D., Kaliva, M., Costanzo, S., Huang, Q., Lutz, P. J., Ahn, J., Chang, T., Rubinstein, M., \& Vlassopoulos, D. (2021). Nonlinear rheometry of entangled polymeric rings and ring-linear blends. Journal of Rheology, 65(4), 695-711. https://doi.org/10.1122/8.0000186

\section{General rights}

Copyright and moral rights for the publications made accessible in the public portal are retained by the authors and/or other copyright owners and it is a condition of accessing publications that users recognise and abide by the legal requirements associated with these rights.

- Users may download and print one copy of any publication from the public portal for the purpose of private study or research.

- You may not further distribute the material or use it for any profit-making activity or commercial gain

- You may freely distribute the URL identifying the publication in the public portal 


\title{
Nonlinear rheometry of entangled polymeric rings and ring-linear blends
}

\author{
Daniele Parisi, ${ }^{1,2}$ Maria Kaliva, ${ }^{1,2}$ Salvatore Costanzo, ${ }^{3}$ Qian Huang, ${ }^{4}$ Pierre J. Lutz, ${ }^{5}$ Junyoung \\ Ahn, ${ }^{6}$ Taihyun Chang, ${ }^{6}$ Michael Rubinstein ${ }^{7}$ and Dimitris Vlassopoulos ${ }^{1,2}$
}

${ }^{1}$ Institute of Electronic Structure \& Laser, Foundation for Research and Technology Hellas (FORTH), Heraklion, Crete 70013, Greece.

${ }^{2}$ Department of Materials Science \& Technology, University of Crete, Heraklion, Crete 71003, Greece

${ }^{3}$ Department of Chemical, Materials, and Production Engineering, Federico II University, 80125 Naples, Italy

${ }^{4}$ Department of Chemical and Biochemical Engineering, Technical University of Denmark 2800 Kgs. Lyngby, Denmark

${ }^{5}$ Institut Charles Sadron, CNRS UPR 22, University of Strasbourg, 67034, Strasbourg, France

${ }^{6}$ Division of Advanced Materials Science and Department of Chemistry, Pohang University of Science \& Technology, Pohang 37673, Korea

7 Departments of Mechanical Engineering and Materials Science, Biomedical Engineering, Chemistry, and Physics, Duke University, Durham, NC 27708, USA

\begin{abstract}
We present a comprehensive experimental rheological dataset for purified entangled ring polystyrenes and their blends with linear chains in nonlinear shear and elongation. In particular, data for shear stress growth coefficient, steady-state shear viscosity, and first and second normal stress differences are obtained and discussed as functions of shear rate as well as molecular parameters (molar mass, blend composition and decreasing molar mass of linear component in blend). Over the extended parameter range investigated, rings do not exhibit clear transient undershoot in shear, in contrast to their linear counterparts and ring-linear blends. For the latter, the size of the undershoot and respective strain appear to increase with shear rate. Universal scaling of strain at overshoot and fractional overshoot (ratio of maximum to steady-state shear stress growth coefficient) indicates subtle differences in the shear-rate dependence between rings and linear polymers or their blends.
\end{abstract}


The shear thinning behaviour of pure rings yields a slope nearly identical to predictions (-4/7) of a recent shear slit model and molecular dynamics simulations. Data for the second normal stress difference are reported for rings and ring-linear blends. While $N_{2}$ is negative and its absolute value stays below that of $N_{1}$, as for linear polymers, the ratio $-N_{2} / N_{1}$ is unambiguously larger for rings compared to linear polymer solutions with the same number of entanglements (almost by factor of two), in agreement with recent non-equilibrium molecular dynamics simulations. Further, $-\mathrm{N}_{2}$ exhibits slightly weaker shear rate dependence compared to $N_{l}$ at high rates, and the respective powerlaw exponents can be rationalized in view of the slit model (3/7) and simulations (0.6), although further work is needed to unravel the molecular original of the observed behaviour. The comparison of shear and elongational stress growth coefficients for blends reflects the effect of ring-linear threading which leads to significant viscosity enhancement in elongation. Along the same lines, the elongational stress is much larger than the first normal stress in shear, and their ratio is much larger for rings and ring-linear blends compared to linear polymers. This confirms the interlocking scenario of rings and their important role in mechanically reinforcing linear matrices.

\section{Introduction}

The rheology of entangled ring polymers has emerged as a topic at the forefront of scientific research. The reasons are the intriguing properties of these materials, which depart from their unlinked counterparts, and the potential applications of rings as elements for tailoring the flow properties of soft materials. Among the many manifestations of the uniqueness of non-concatenated entangled rings, we mention their power-law stress relaxation [1,2], their weaker shear thinning of viscosity compared to their linear counterparts [2,3], their role in enhancing the viscosity of linear polymers [4-7] and their strong resistance to stretching (strain hardening at low stretch rates) [8-10]. These unusual phenomena can be explained by the loopy conformations of the rings which yield self-similar relaxation, threading by linear chains and ring interlocking in an elongated melt, respectively. In a remarkable synergy of advanced characterization, rheometry, simulations and modeling, the field has thrived in the last decade and continues doing so, while several open questions remain. Clearly, for a deep understanding of rheology of entangled ring polymers, there is a need to obtain more and reliable experimental evidence of the response of ring polymers in nonlinear shear and elongational flows. In particular, recent work with experimentally pure polystyrene rings having about $Z=5$ and 12 entanglements indicates that they exhibit much weaker shear thinning compared to their linear counterparts [3,11]. Furthermore, the specific viscosity enhancement of entangled linear polymers upon addition of small fractions of rings was explained by invoking the constraint release process of 
the rings [4], whereas these blends were shown to exhibit significant strain hardening in uniaxial extension and an overshoot in the elongational stress growth coefficient which was associated to a transient threading-unthreading transition [8]. The above exciting results open new routes in molecular rheology and particularly in the direction of combining the features of rings and other macromolecular architectures to tune the viscoelastic properties of polymeric materials. The available evidence must be complemented by systematic experiments with different molar masses, fraction of ring component in blends, blend asymmetry (ratio of components molar masses) and, eventually, different chemistry (and flexibility). Importantly however, addressing the first normal stress difference $\left(\mathrm{N}_{1}\right)$, as well as the much-neglected second normal stress difference $\left(\mathrm{N}_{2}\right)$, in entangled rings is timely. Indeed, recent advances have facilitated the experimental investigations in this direction which, nevertheless, remain very challenging. However, we have shown recently that measuring all three material functions in high-temperature melts is possible by appropriate use of a modular cone-partitioned plate (CPP) setup [12].

It should be noted that progress in simulations has been remarkable as well. The weaker shear thinning of rings compared to their linear counterpart, their nonlinear response at low elongational rates accompanied by extension rate thickening and ring-ring interlocking, and the threading-unthreading transition represent the punchline of the rich outcome so far [8,9,13-17]. Very recently, the $\mathrm{N}_{1}$ and $\mathrm{N}_{2}$ of moderately entangled poly(ethylene oxide) rings were investigated by means of atomistic molecular dynamics simulations and it was found that the ratio $-\mathrm{N}_{2} / \mathrm{N}_{1}$ is clearly larger compared to linear polymers [16]. On the modeling part, the shear thinning of viscosity has been shown to be captured quantitatively by a new slit model which also explains many details of the conformation of the sheared rings. It is based on the presence of two length scales: the thickness of the shear slit in the velocity gradient direction and a smaller length scale, the tension blob size due to strong stretching of rings in the flow direction. A ring is confined in the velocity gradient direction to the shear slit with thickness $\xi_{s}$, that defines the shear blob with relaxation time on the order of reciprocal shear rate $1 / \dot{\gamma}$. The model further provides scaling predictions for $N_{1}$ and $N_{2}$ reciprocally proportional to these two length scales [11], which are discussed in the context of the experimental results in the present work.

In this work we address the above challenges with one ambitious goal, to provide a comprehensive set of reliable experimental nonlinear rheological data with purified entangled ring polymers and their blends with linear chains. The data involve shear stress growth coefficient, steady-state viscosity and first and second normal stress differences, and comparison with respective elongational data. Importantly, in view of the significant role of the addition of small amounts of rings in altering the properties of linear matrices, as mentioned above, we focus at this range of ring-linear blend 
composition; one advantage is that, here ring purification is not as crucial as in the study of the individual ring component or blends with large ring fraction (which are not addressed here). We discuss the delicate points and possible intricacies of the experiments, the role of blend composition, the behavior of $\mathrm{N}_{1}$ and $\mathrm{N}_{2}$ and the link to existing information from simulations, while we identify new challenges ahead.

\section{Materials and Methods}

This study is based on measurements with thirteen polystyrene (PS) samples, including pure components and blends. In particular, two linear polymers (L185, L130), and three purified rings (R185, R90, R30), covering the range from onset of entanglements (R30) to well-entangled (R185), along with. five symmetric linear/ring and three asymmetric linear/ring PS blends with smaller molar mass of the linear component, were investigated. The samples were carefully characterized and R185 and R90 were purified by liquid chromatography at critical condition, (LCCC); see Fig. S1 of the Supporting Information (SI). The main molecular characteristics of the samples are listed in Table 1. Sample coding reflects the molecular architecture, linear (L) and/or ring (R), followed by a number identifying the weight-average molar mass $\left(\mathrm{M}_{\mathrm{w}}\right)$ of the polymer, expressed in $\mathrm{kg} / \mathrm{mol}$; the latter is often abbreviated as $(\mathrm{k})$. The details of the synthesis, purification, and characterization schemes of R185 are described elsewhere [18-21]. The linear PS L90 was synthesized using potassium naphthalenide as initiator in tetrahydrofuran (THF), while the ring R90 was obtained by coupling the bifunctional anionic PS with $\alpha, \alpha$ 'dibromo- $p$-xylene in very dilute THF solution [22,23]. Same procedure was followed for R30. After standard fractionation with benzene methanol [24], the R90 ring was further purified and characterized by a procedure similar to that followed for R185 [1,4,9]. Purification of as-prepared PS and characterization confirming the purity of the R90 are presented in Fig. S1 of the Supplemental Information (SI). Sample R30 was undergone multiple SEC runs (Fig.S2, [24]) and the final product had an acceptable level of purity as judged by the combination of the final chromatogram and its linear rheology (discussed below). Blends are characterized by the weight fraction of rings, $\phi_{\mathrm{R}}$.

The Rouse, $\tau_{\mathrm{R}}$, the terminal (disentanglement), $\tau_{\mathrm{d}}$, and the ring terminal, $\tau_{\mathrm{Ring}}$, times of the pure components are reported in Table 2 at $160{ }^{\circ} \mathrm{C}$. The relaxation times have been extracted from the linear viscoelastic master curves, in terms of storage $\mathrm{G}^{\prime}$ and loss modulus $\mathrm{G}^{\prime}$, obtained through small amplitude oscillatory shear experiments at various temperatures. The inverse high-frequency crossover is assigned to the Rouse relaxation time of an entanglement strand, $\tau_{\mathrm{e}}[4,8]$. For polystyrene linear polymer chains $\tau_{\mathrm{e}}$ is $0.002 \mathrm{~s}$ at $150{ }^{\circ} \mathrm{C}$ [4]. Times at various temperatures have been calculated 
by using the horizontal shift factor reported in Parisi et al. [4]. The Rouse time for linear polymer chains has been estimated as $\tau_{\mathrm{R}}=\tau_{\mathrm{e}}\left(\mathrm{N} / \mathrm{N}_{\mathrm{e}}\right)^{2}[25,26]$ where $\mathrm{N}$ and $\mathrm{N}_{\mathrm{e}}$ are the numbers of Kuhn monomers per chain and entanglement strand, respectively. $\mathrm{N}_{\mathrm{e}}$ has been estimated as the ratio between the entanglement molar mass of PS, $\mathrm{Me}_{\mathrm{e}}=17.5 \mathrm{~kg} / \mathrm{mol}$ [27] and the respective molar mass of a polystyrene Kuhn segment, $\mathrm{M}_{0}=0.72 \mathrm{~kg} / \mathrm{mol}$ [24]. The number of entanglements of the pure components (linear and ring polymers) and blends is denoted as Z. Values are reported in Table 1. Note that the Rouse time of ring polymers has been estimated as $1 / 4$ of that of linear polymers with the same molar mass [28-30]. The terminal times are estimated from the inverse frequency at the crossover of the extrapolated terminal slopes of the dynamic moduli $[4,9]$.

Table 1. Ring, linear and ring-linear blend samples investigated and their key characteristics.

\begin{tabular}{llccc}
\hline Sample code & Components & $\mathbf{M}_{\mathbf{w}}(\mathbf{k g} / \mathbf{m o l}){ }^{*}$ & Notes & $\mathbf{Z}^{* *}$ \\
\hline L185 & PS Linear 185k & 185 & Linear & 10.6 \\
L130 & PS Linear 130k & 133 & Linear & 7.4 \\
R185 & PS Ring 185k & 185 & Ring & 10.6 \\
R90 & PS Ring 90k & 89.2 & Ring & 5.1 \\
R30 & PS Ring 30k & 30 & Ring & 1.7 \\
\hline & $\phi_{\mathrm{R}}=0.05$ & & & 10.6 \\
L185/R185 & $\phi_{\mathrm{R}}=0.15^{* *}$ & & Symmetric & 10.6 \\
& $\phi_{\mathrm{R}}=0.2$ & Linear/Ring & 10.6 \\
& $\phi_{\mathrm{R}}=0.3$ & & & 10.6 \\
L90/R90 & $\phi_{\mathrm{R}}=0.72^{* * *}$ & & Asymmetric & 7.1 \\
\hline \multirow{2}{*}{ L130/R185 } & $\phi_{\mathrm{R}}=0.05$ & & Linear/Ring & 7.4 \\
& $\phi_{\mathrm{R}}=0.2$ & & & 7.4 \\
& $\phi_{\mathrm{R}}=0.3$ & & & 7.4 \\
\hline \hline
\end{tabular}

* measured by light scattering, except for the molar mass of R30 which was determined based on PS standards.

** based on literature value for $\mathrm{M}_{\mathrm{e}}$ and considering that rings and linear polymers have the same $\mathrm{M}_{\mathrm{e}}$. For asymmetric blends it is averaged based on the weight fraction of the components.

${ }^{* * *}$ used only for normal stress measurements.

Table 2. Characteristic relaxation times of the pure components at $160{ }^{\circ} \mathrm{C}$.

\begin{tabular}{lcc}
\hline Sample code & $\tau_{\mathbf{R}}[\mathbf{s}]$ & $\tau_{\mathbf{d}}, \tau_{\text {Ring }}[\mathbf{s}]$ \\
\hline L185 & 0.051 & 2.940 \\
L130 & 0.025 & 0.500 \\
R185 & 0.013 & 0.138 \\
R90 & $0.003^{\#}$ & 0.03 \\
R30 & 0.00034 & 0.008 \\
\hline \hline \multirow{2}{*}{ \# Estimated as $\frac{\tau_{R, L 185 k}}{4}\left(\frac{Z_{90 k}}{Z_{185 k}}\right)^{2}$, with $\tau_{R, L 185 k}=0.051, Z_{90 k}=5.14$, and $Z_{185 k}=10.6}$.
\end{tabular}

Blends were prepared by mixing specified amounts of rings and linear polymers (based on $\phi_{\mathrm{R}}$ ) in toluene in the dilute regime. Solutions were kept at room temperature for about two days and gently stirred at times in order to ensure complete dispersion. Subsequently, toluene was removed under 
vacuum over 7 days, and the resulting blends were press-molded into disks at $150{ }^{\circ} \mathrm{C}$ (well above the glass transition temperature) for twenty minutes under vacuum to reduce thermal oxidation. The same press-molding procedure was adopted for the pure components.

\subsection{Shear Rheology}

Shear rheology experiments were performed on a rotational strain-controlled ARES rheometer (TA, USA) equipped with a force rebalance transducer 2KFRTN1. The temperature was controlled by means of a convection oven with an accuracy of $\pm 0.1{ }^{\circ} \mathrm{C}$ and fed with nitrogen gas to minimize the risk of thermal degradation of the samples. As the investigated polymer melts are prone to edge fracture, a cone-partitioned plate (CPP) geometry was used for all the nonlinear shear experiments. Details about this geometry are reported elsewhere [3,12]. Here, a stainless steel $25 \mathrm{~mm}$ diameter cone with truncation equal to $0.051 \mathrm{~mm}$, and cone angle of $0.1 \mathrm{rad}$ was used. Various diameters for the inner partition of the CPP were used; $2,3,4$, and $6 \mathrm{~mm}$, as specified in the text below. Pressmolded disks were loaded by using a centering tool, which ensures the proper centering of the sample in the measuring area. Linear viscoelastic master curves for L130, R90 and L130/R185 blends were obtained with stainless steel $8 \mathrm{~mm}$ diameter parallel plates at various temperatures in the range 120 $160{ }^{\circ} \mathrm{C}$. Master curves for L185, R185 and L185/R185 blends were taken from the literature [4]. The horizontal and vertical shift factors were taken from Parisi et al. [4].

The technique reported by Costanzo et al. [12] to extract the first $\mathrm{N}_{1}$ and second $\mathrm{N}_{2}$ normal stress differences was adopted. Briefly, the method consists of performing start-up of shear rate experiments at various shear rates, by using the CPP. The same experiments are performed with different inner partition diameters. This allows us to determine the apparent first normal stress difference $\left(\mathrm{N}_{1, \mathrm{app}}\right)$ at different diameters but same shear rates. For instance, $\mathrm{N}_{1 \text {,app }}$ corresponds to the apparent first normal stress difference measured with a CPP having an inner partition of radius $\mathrm{R}_{\text {inner. }}$ In the present case, we used two different inner partitions per each shear rate, with radii $R_{\text {inner,i }}$ and $R_{\text {inner,ii. The relations }}$ that correlate $\mathrm{N}_{1, \text { app,i, }} \mathrm{N}_{1, \text { app,ii, }} \mathrm{N}_{1}$, and $\mathrm{N}_{2}$ are [12]:

$$
N_{1, a p p, i}=\frac{2 F_{i}}{\pi R_{1}^{2}}=N_{1}+2\left(N_{1}+2 N_{2}\right) \ln \left(\frac{R}{R_{\text {inner }, i}}\right)
$$




$$
N_{1, a p p, i i}=\frac{2 F_{i i}}{\pi R_{2}^{2}}=N_{1}+2\left(N_{1}+2 N_{2}\right) \ln \left(\frac{R}{R_{\text {inner }, i i}}\right)
$$

$\mathrm{F}_{\mathrm{i}}$ and $\mathrm{F}_{\mathrm{ii}}$ are the normal forces exerted by the sample when the inner partition Rinner,i or Rinner,ii is used, respectively; $\mathrm{R}$ is the radius of the sample loaded in the measuring position, estimated as $[12,31,32]$ :

$$
R=\left(\frac{3 m}{2 \pi \rho \theta}\right)^{1 / 3}
$$

where $m$ is the sample mass, $\rho$ the sample density $\left(0.995 \mathrm{~g} / \mathrm{cm}^{3}\right.$ for PS at $\left.150{ }^{\circ} \mathrm{C}\right)[33]$ and $\theta$ the cone angle expressed in rad. By using a sample mass between 8 and $23 \mathrm{mg}$, we obtain $\mathrm{R}$ values between

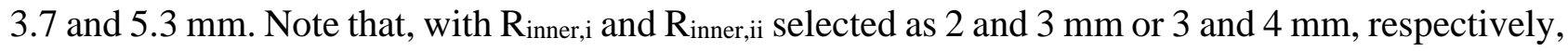
the same sample mass was used. However, the latter is not a necessary condition to satisfy Eqs. 1-2 $[12,32]$. The two normal stress differences $\mathrm{N}_{1}$ and $\mathrm{N}_{2}$ can be extracted by solving the system of Eqs. 1-2, where $\mathrm{R}$ is calculated according to Eq. 3. In order to ensure the same treatment of samples and reduce concerns of possible fracture and sample accumulation in the gap between plate and partition, prior to applying a shear flow, a fresh disk was used for each shear rate. Finally, a note on the apparent normal stress is in order. The above approach is crucially important for extracting $\mathrm{N}_{2}$ whose value is much smaller than that of $\mathrm{N}_{1}$ (see discussion below). Indeed, by considering the different radii of geometries and samples used, the ratio $-\mathrm{N}_{2} / \mathrm{N}_{1}$ (not exceeding 0.5) and the weak shear rate dependence of the normal stress differences (see also discussion below), we estimate from Eq.1 (or Eq.2) that the ratio $\mathrm{N}_{1, \text { app }} / \mathrm{N}_{1}$ varied between 1.15 and 1.75. In fact, the lowest and highest values were attained for L185 and R185, respectively (note however that the former is the only sample for

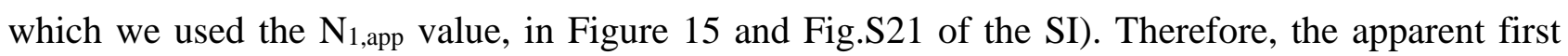
normal stress difference $\mathrm{N}_{1 \text {,app }}$ represents a reasonable estimation of the first normal stress difference, as will be further discussed below [12,32-35].

\subsection{Extensional Rheology}

The extensional stress measurements were performed using a filament stretching rheometer (FSR) [36]. Prior to performing a measurement, the samples were molded into cylindrical test specimens with a fixed radius of either $R_{0}=2.7 \mathrm{~mm}$ or $R_{0}=4.0 \mathrm{~mm}$. The aspect ratio $\Lambda_{0}=L_{0} / R_{0}$, with $L_{0}$ being the initial length, was between 0.42 and 0.64 . The smaller radius and lower aspect ratio were used due to the limited amount of sample. The samples were press-molded following the same 
aforementioned procedure. All the samples were pre-stretched to a radius $R_{p}$ ranging from 1.2 to 3 $\mathrm{mm}$ at $150-160{ }^{\circ} \mathrm{C}$ prior to the extensional experiments. After pre-stretching, the temperature was decreased to $T=130{ }^{\circ} \mathrm{C}$ for the extensional stress measurements. A few measurements were performed at $150{ }^{\circ} \mathrm{C}$ or $160{ }^{\circ} \mathrm{C}$, and shifted to $130{ }^{\circ} \mathrm{C}$ by using the shift factors reported in Ref. [4]. Nitrogen gas atmosphere was ensured during the whole test.

During the extensional measurements, the force $F(t)$ was measured by a load cell and the diameter $2 R(t)$ at the mid-filament plane was measured by means of a laser micrometer. At small deformations during the startup of elongational flow, part of the stress difference arises from the radial variation due to the shear components in the deformation field. This effect may be compensated by a correction factor as described elsewhere [37]. For large deformations, the correction vanishes and the radial variation of the stress in the symmetry plane becomes negligible [38].

The Hencky strain $\varepsilon(t)$ and the mean value of the stress difference $\left\langle\sigma_{z z}-\sigma_{r r}\right\rangle$ over the mid-filament plane are calculated from observations of $R(t)$ and $F(t)$ as:

$$
\begin{gathered}
\varepsilon(t)=-2 \ln \left(R(t) / R_{p}\right) \\
\left\langle\sigma_{z z}-\sigma_{r r}\right\rangle=\frac{F(t)-m_{f} g / 2}{\pi R(t)^{2}} \cdot \frac{1}{1+\left(R(t) / R_{0}\right)^{10 / 3} \cdot \exp \left(-\Lambda_{0}^{3}\right) /\left(3 \Lambda_{0}^{2}\right)}
\end{gathered}
$$

where $m_{f}$ is the weight of the filament and $g$ is the gravitational acceleration. The Hencky strain rate is defined as $\dot{\epsilon}=\mathrm{d} \epsilon / \mathrm{d} t$. A control scheme [39] is employed in the FSR to ensure accurate constant strain rate. The extensional stress growth coefficient is defined as $\bar{\eta}^{+}=\left\langle\sigma_{z z}-\sigma_{r r}\right\rangle / \dot{\epsilon}$.

\section{Results and Discussion}

\section{Linear viscoelastic characterization.}

Figure 1 depicts the linear viscoelastic (LVE) master curves $\left(\mathrm{G}^{\prime}\right.$ in panel $A$ and $\mathrm{G}^{\prime \prime}$ panel $\mathrm{B}$ ) for rings R185, R90, and R30, linear L185, L130 and blends L185/R185, L130/R185 and L90/R90, at a reference temperature of $160^{\circ} \mathrm{C}$. This sets the necessary background for the present study: it confirms the power-law stress relaxation of the purified rings and at the same time the quality of the samples. In fact, the lines through the (pure) ring data represent fits with the state-of-the-art fractal loopy globule (FLG) model which predicts a power-law exponent of 3/7 [34]. Actually, for R30 which was multiply fractionated by standard SEC and corresponds to transition to entanglement regime $(Z=1.7)$, 
the data are well-described by the model but we also note that the linear precursor does not exhibit a plateau, hence our assessment of the sample's quality is based on the extracted value of the zero-rate viscosity (Figure 2 below). The latter is slightly higher than expected, suggesting that this ring is not as pure as the others (which have been fractionated by LCCC) but is appropriate for use in the rheometric study discussed in subsequent sections. The low-frequency deviation is attributed to remaining traces of unlinked linear and possible effects of long-living ring-ring threading [14], but overall, it remains a debatable issue which will not be discussed further. In addition, the data of Figure 1 confirm that the blends studied here (with fraction of the linear component being 0.7 or higher) are characterized by the presence of the rubbery plateau region. Details of their relaxation via constraint release due to linear motion are discussed in Ref. [4], and more recently in Ref. [40]. Figure 2 presents the zero-shear viscosity data for the pure components of Figure 1 at the same reference temperature of $160^{\circ} \mathrm{C}$. The values were obtained from the complex viscosity, extracted from the lowest measured frequencies in the Newtonian regime. Data from the literature on PS of different molar masses have been added and are in excellent agreement. The slight scattering of PS ring data likely reflects different level of purity (which remains always high) as discussed in the literature [1,18-20,24,41]. Of particular note is the striking difference in the $\mathrm{M}_{\mathrm{w}}$ dependence of $\eta_{0}$ between linear and ring polymers which conforms to predictions and confirms and expands earlier data with purified rings and different chemistries [42]. There is a notable, albeit small, deviation of the data for R30, as well as for the higher molar masses. In the former case, the level of purity seems to be not as high as in the other samples, as already mentioned. In the latter case, despite the fact that even LCCC fractionation is not perfect, the ultra-small amount of samples renders their continuous use and thermal treatment necessary, suggesting the possibility of some contamination due to unlinking of some rings. Whereas we do not know when this may happen during a measurement cycle, in an extreme case of overused sample, we checked the linear spectra and its SEC trace and found differences [43]. For the presented data, the reproducibility of the linear viscoelastic data served as indicator of the good quality of the samples. 


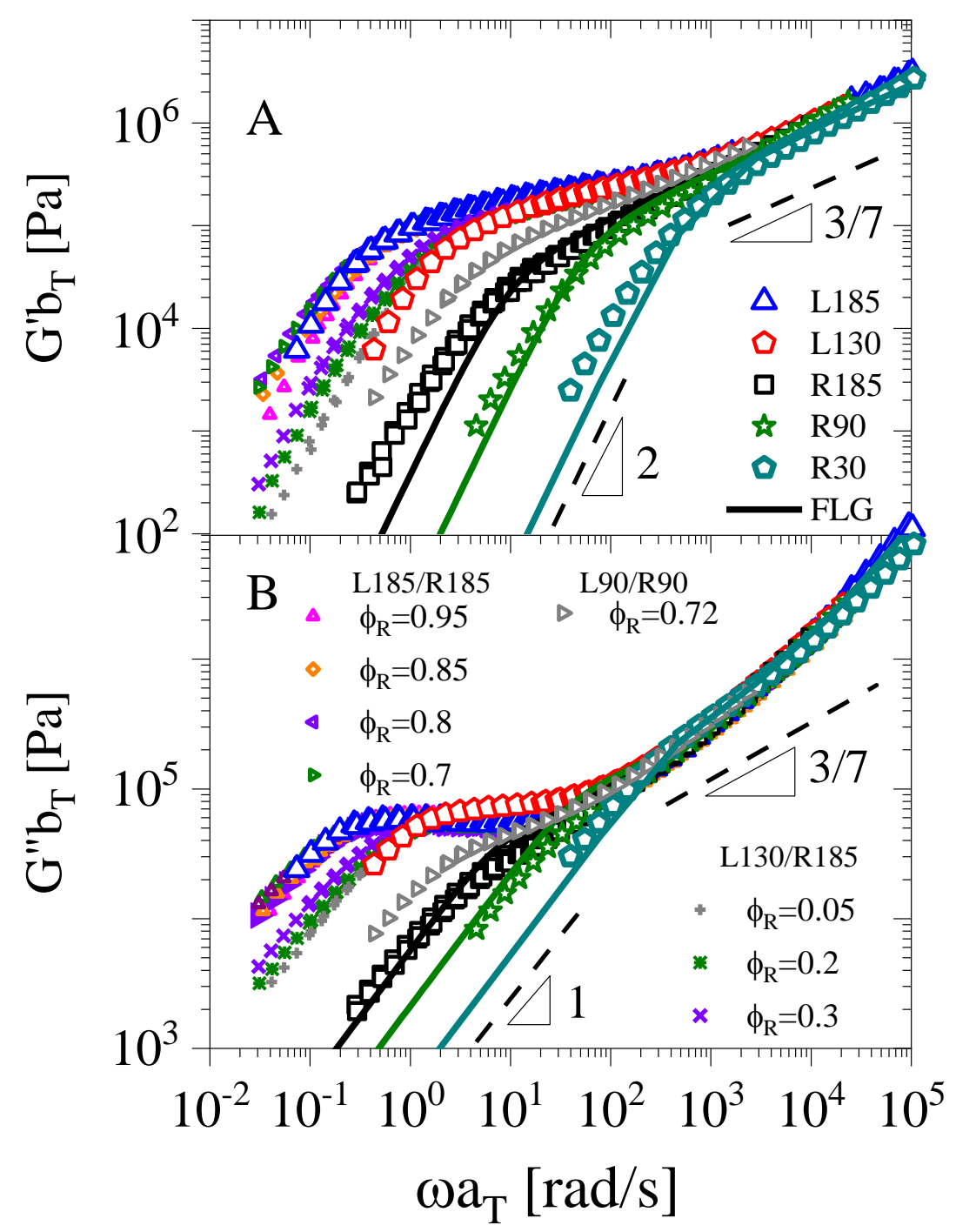

Figure 1. Linear viscoelastic master curves of storage $\mathrm{G}^{\prime}$ (panel A) and loss G" (panel B) moduli as a function of oscillatory frequency for pure components and blends. Details are reported in the legend. The reference temperature is $160{ }^{\circ} \mathrm{C}$. Dashed lines with slopes 1 and 2 indicate the terminal slopes of the moduli. The solid lines represent the prediction of the Fractal Loopy Globule (FLG) model for ring polymers [44], with a 3/7 power-law exponent for the modulus (dashed black line). Data for PS Linear 185, Ring 185 and their blends are taken from Ref. [4]. 


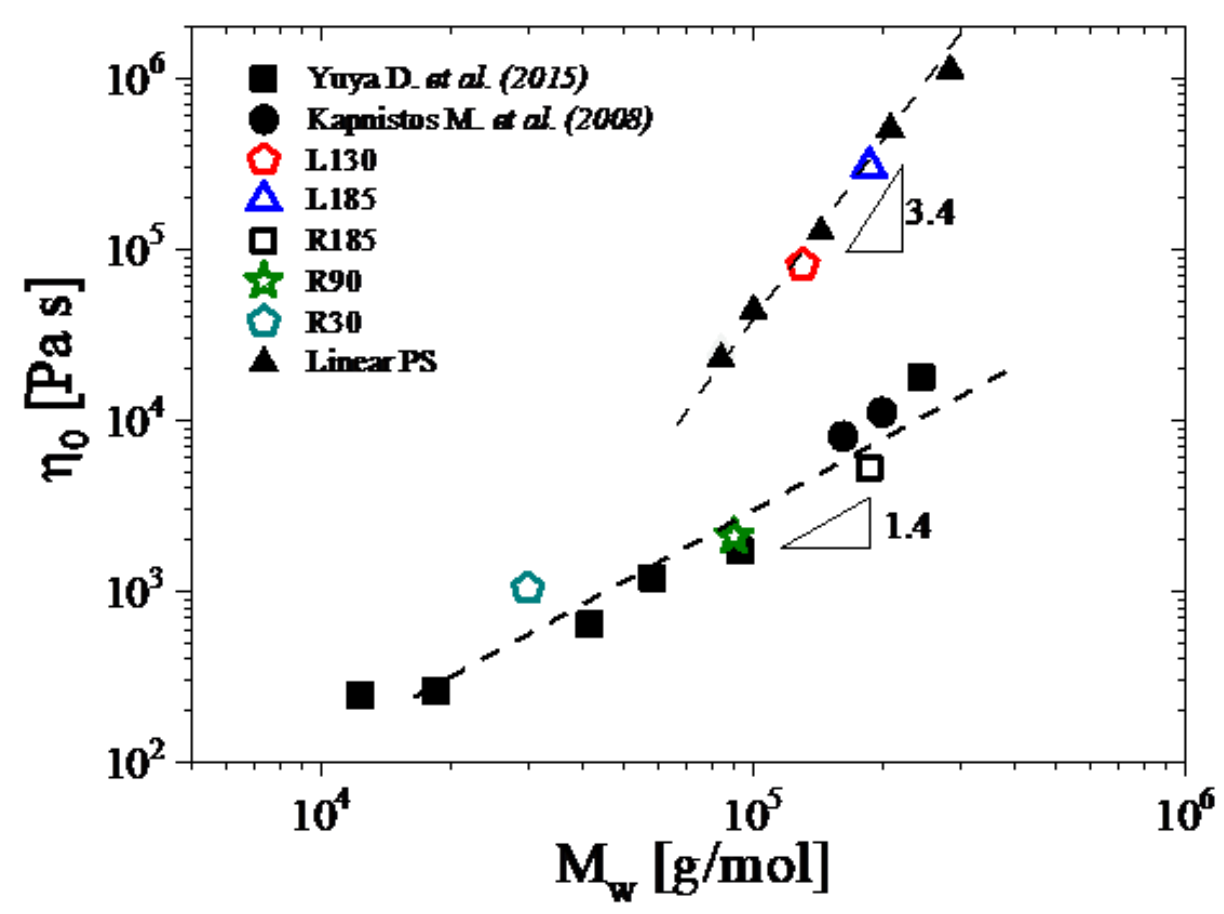

Figure 2. Zero-shear rate viscosity $\eta_{0}$ as a function of the weight-average molar mass $\mathrm{M}_{\mathrm{w}}$ for the pure components reported in Figure 1. Additional linear PS data (solid triangles) are also reported together with high-purity ring PS from the literature $[1,28]$. All the data are reported at $160{ }^{\circ} \mathrm{C}$. The dashed lines represent the scaling law expected for entangled linear polymer melts with exponent 3.4 [25], and for ring polymers with exponent 1.4 [44].

\section{Transient shear response.}

In Figure 3 we show typical results of start-up of shear experiments in the form of shear stress growth coefficient at different imposed shear rates for the two linear polymers L185 and L130 and for two ring polymersR185 and R90 (the shear stress growth coefficient for R30 is shown in Figure S13 of the SI). The range of Rouse-Weissenberg numbers $\left(W i_{R}=\dot{\gamma} \tau_{R}\right)$ explored is reported in Table 3 for the systems investigated in this work. The lower values of $\mathrm{Wi}_{\mathrm{R}}$ are often within linear regime leading to the zero-shear-rate viscosity ( $\left.\eta_{0}\right)$ at long times, whereas values larger than one correspond to the stretched chain regime. Similarly, it is possible to estimate the terminal-Weissenberg numbers as $W i_{d}=\dot{\gamma} \tau_{d}$ and $W i_{\text {Ring }}=\dot{\gamma} \tau_{\text {Ring }}$ for linear chains, blends, and for pure rings, respectively. 
Table 3. Wir range in start-up of steady shear experiments.

\begin{tabular}{lc}
\hline Sample code & Wi $_{\mathrm{R}}$ range \\
\hline $\mathrm{L} 185$ & $0.005-1.6$ \\
$\mathrm{~L} 130$ & $0.0025-2.5$ \\
$\mathrm{R} 185$ & $0.01-3.33$ \\
$\mathrm{R} 90$ & $0.04-4.0$ \\
$\mathrm{R} 30$ & $0.0027-0.35$ \\
$\mathrm{~L} 185 / \mathrm{R} 185 \phi_{\mathrm{R}}=0.05$ & $0.0016-0.29$ \\
$\mathrm{~L} 185 / \mathrm{R} 185 \phi_{\mathrm{R}}=0.2$ & $0.0016-2.0$ \\
$\mathrm{~L} 185 / \mathrm{R} 185 \phi_{\mathrm{R}}=0.3$ & $0.0005-2.0$ \\
$\mathrm{~L} 130 / \mathrm{R} 185 \phi_{\mathrm{R}}=0.05$ & $0.0008-1.4$ \\
$\mathrm{~L} 130 / \mathrm{R} 185 \phi_{\mathrm{R}}=0.2$ & $0.0008-1.4$ \\
$\mathrm{~L} 130 / \mathrm{R} 185 \phi_{\mathrm{R}}=0.3$ & $0.0008-0.8$ \\
\hline \hline
\end{tabular}

All other transient data are presented in the SI, Figs.S3-S13. It is encouraging that the respective LVE envelope, as deduced from the respective frequency spectra of Fig. 1 and transformed into shear stress growth coefficient by means of the Cox-Merz and Gleissle $[34,45]$ rules, match the low-shear-rate start-up data. Further, the fact that steady-state is reached for shear rates covering more than two decades is a testimony of the present experimental ability to study the nonlinear rheology with this type of setup reliably. The steady-state values of the shear stress growth coefficient decrease with increasing shear rate (see further discussion below). We observe however some differences in the time evolution of the shear stress growth coefficient of linear and ring polymers, and particularly in the regime between overshoot and steady state. The linear polymers exhibit a clear undershoot at high shear rates (Fig.3A,B). However, this is not the case for the rings of the same molar mass, for which no undershoot is observed over the range of investigated shear rates (Fig.3C,D). Moreover, at the highest rates the sample fractures before the steady-state value is attained (data not shown). This points to a clear difference in the shear-induced conformational changes in the two macromolecular architectures. Before further discussing the features of undershoot, we confirm that based on careful experiments (see references $[12,46]$ and the present data) it is real. We also note that recent slip-link simulations show that undershoot is not linked to slip (which tends to suppress it) [47]. Recently, conformational differences between extended single DNA ring and linear polymers have been reported in the context of coil-stretch transition [47]. Importantly, the undershoot (or minimum in the transient response) of linear polymers has been linked to transient tumbling [34,49]; if undershoots 
occur in rings, this should happen at higher rates which are not accessible experimentally at the moment. The conjecture of different conformation in sheared ring and linear polymers is supported by recent insightful evidence about their response in dilute or non-dilute solution from both experiments $[10,50]$ and simulations [51-53], as well as simulations and modeling in the melt state $[11,13-16]$.
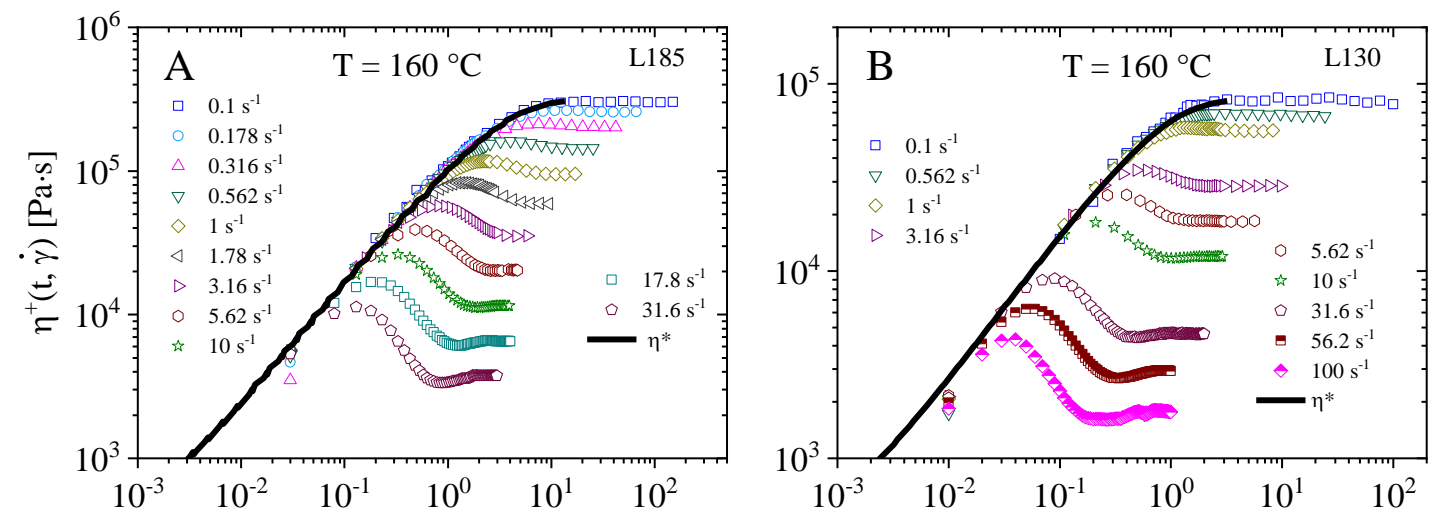

$\mathrm{t}[\mathrm{s}]$
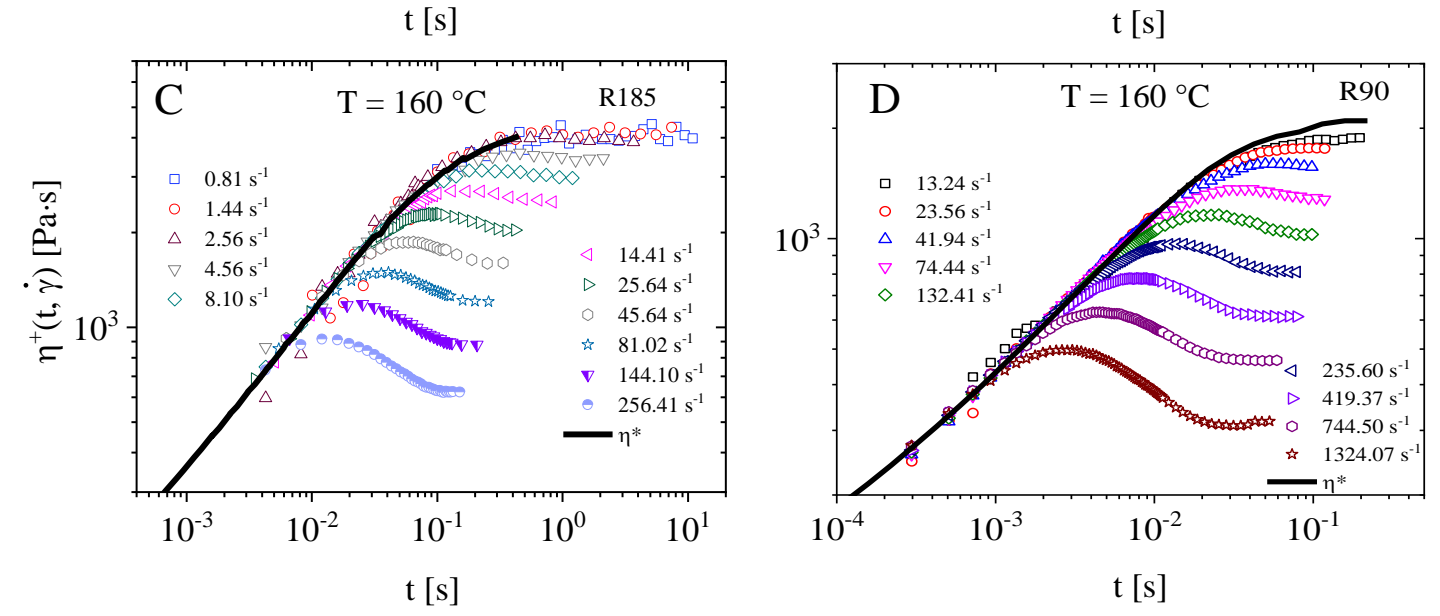

Figure 3. Shear rate start-up in terms of shear stress growth coefficient as a function of time at various shear rates (reported in the legend) for A) L185, B) L130, C) R185, and D) R90. Experimental data refer to the same temperature, $160^{\circ} \mathrm{C}$. The sample R90 was measured at $130{ }^{\circ} \mathrm{C}$ and data were shifted to $160{ }^{\circ} \mathrm{C}$ by using the shift factors reported in Ref. [4]. The R30 sample is reported in Figure S13 of the SI. Black solid lines report the LVE envelope, i.e., the complex viscosity $\left(\eta^{*}\right)$ as a function of the inverse oscillatory frequency.

Adding rings to linear matrices alters the nonlinear shear response in a subtle way. The message from Fig.4 is that, even with a ring fraction as high as 0.3 , the qualitative features of linear chains are predominant, similar conclusion based on linear viscoelasticity results [1,2,4-6]. For example, the undershoot in the transient response is observed at high shear rates. The undershoot data are presented in Fig.5. The strain at the undershoot minimum, $\gamma_{M I N}$, increases with Weissenberg number (here we 
consider WiR) by a factor of 3 (or factor of 2 for a given sample L185, Fig.5A) over the range of WiR between 0.2 and 3 approximately (range 0.5 to 2 for L185). The magnitude of the fractional undershoot, i.e., the ratio of the minimum to steady-state shear stress growth coefficient, $\eta_{M I N} / \eta_{\text {steady-state }}$, systematic decreases (by about $10 \%$ ) as WiR increases from 0.25 to 3 (Fig.5B). Interestingly, whereas the asymmetric blends exhibit undershoot, this is not the case for the symmetric ones; however the latter were measured at lower rates at which the asymmetric do not exhibit undershoot either (Fig.4). On the other hand, the largest $\mathrm{Wi}_{\mathrm{R}}$ values reached for the rings is similar to (or even slightly larger than) WiR reached for the symmetric blends (see Table 3). Therefore, to assess the effect of decreasing the molar mass of the linear component of the blend there is a need for more investigations with experiments and simulations. Finally, it can be observed from Figs. 3 and 4 that the main features of shear thinning and the matching of the low-shear-rate data with the LVE envelope are maintained for all examined blend compositions. To better appreciate the shear thinning regime, we present in Figure 6 the flow curves normalized by the zero-shear viscosity and the terminal time, for both pure components (panel A) and blends (panel B). Viscosities from oscillatory and steady shear measurements are plotted and found to validate the Cox-Merz (CM) rule with the exception of a divergence of the latter data from the complex viscosity envelope for the high $\mathrm{M}_{\mathrm{w}}$ linear chains at very high shear rates. From the experimental standpoint, the occurrence of instabilities (primarily the onset of weak wall slip and edge fracture propagating up to the gap of the CPP), cannot be avoided at large terminal Wi numbers (>30), despite the precautions taken. On the other hand, similar deviations were recently reported with simulations and attributed to reduction of the tension blob size [11]. Data are fitted with a modified Carreau-Yasuda equation [11,54-55], $\frac{\eta}{\eta_{0}}=1 /(1+W i)^{m}$, where $m$ is the shear thinning exponent. The latter takes values of 0.89 for pure linear melts, between 0.85 and 0.89 for the blends, and 0.56 for the pure ring polymers, in agreement with the slit model and molecular dynamics simulations presented in Ref. [11]. 


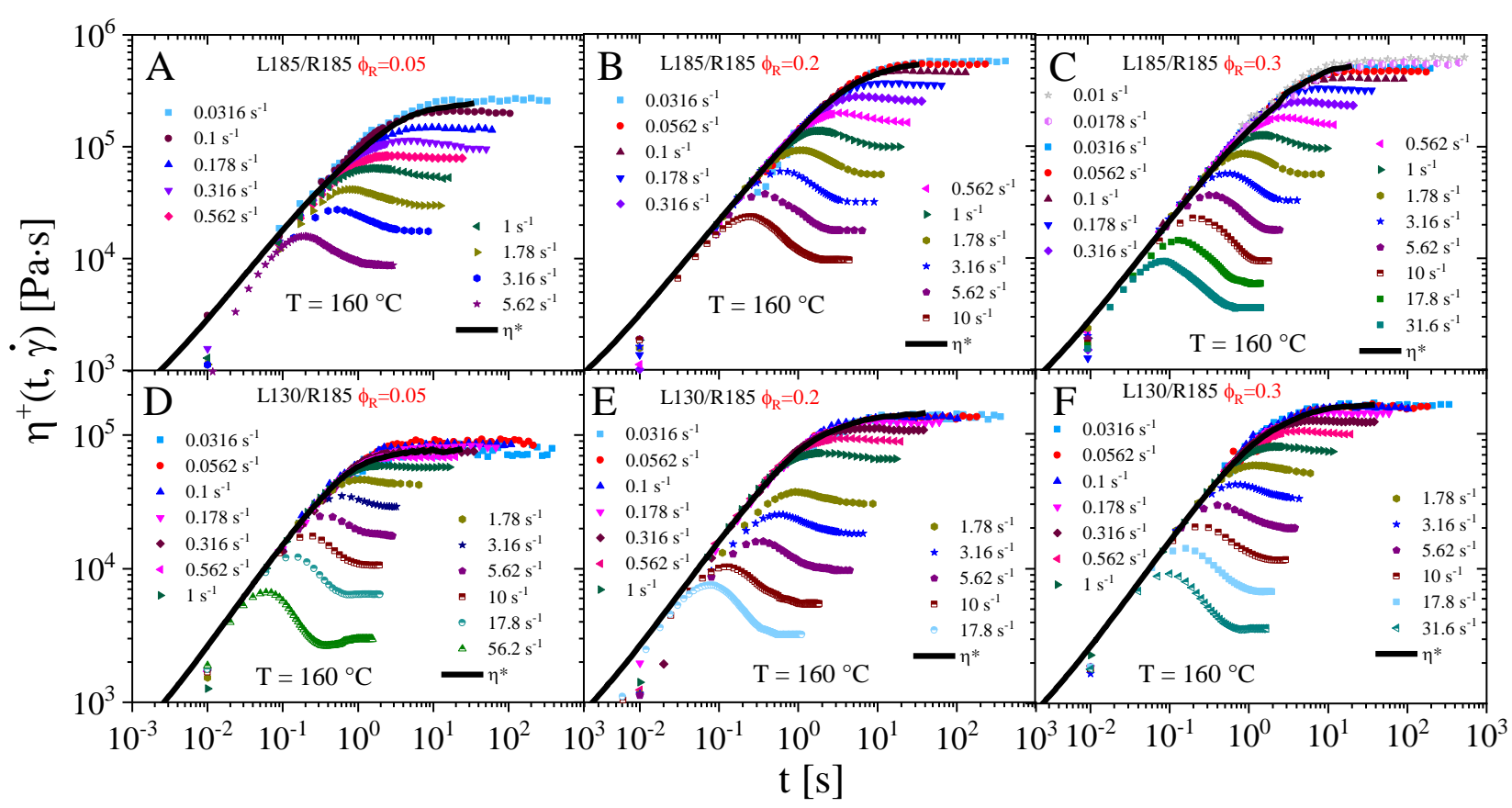

Figure 4. Start-up of shear rate in terms of shear stress growth coefficient as a function of time at various shear rates (reported in the legend) for symmetric blends L185/R185 at A) $\phi R=0.05, \mathrm{~B}$ ) $\phi R$ $=0.2$, and C) $\phi_{\mathrm{R}}=0.3$, and for asymmetric blends L130/R185 at at D) $\left.\phi_{\mathrm{R}}=0.05, \mathrm{E}\right) \phi_{\mathrm{R}}=0.2$, and F) $\phi_{\mathrm{R}}=0.3$. Experimental data refer to the same experimental temperature, $160{ }^{\circ} \mathrm{C}$. Black solid lines report the complex viscosity $\left(\eta^{*}\right)$ as a function of the inverse oscillatory frequency.
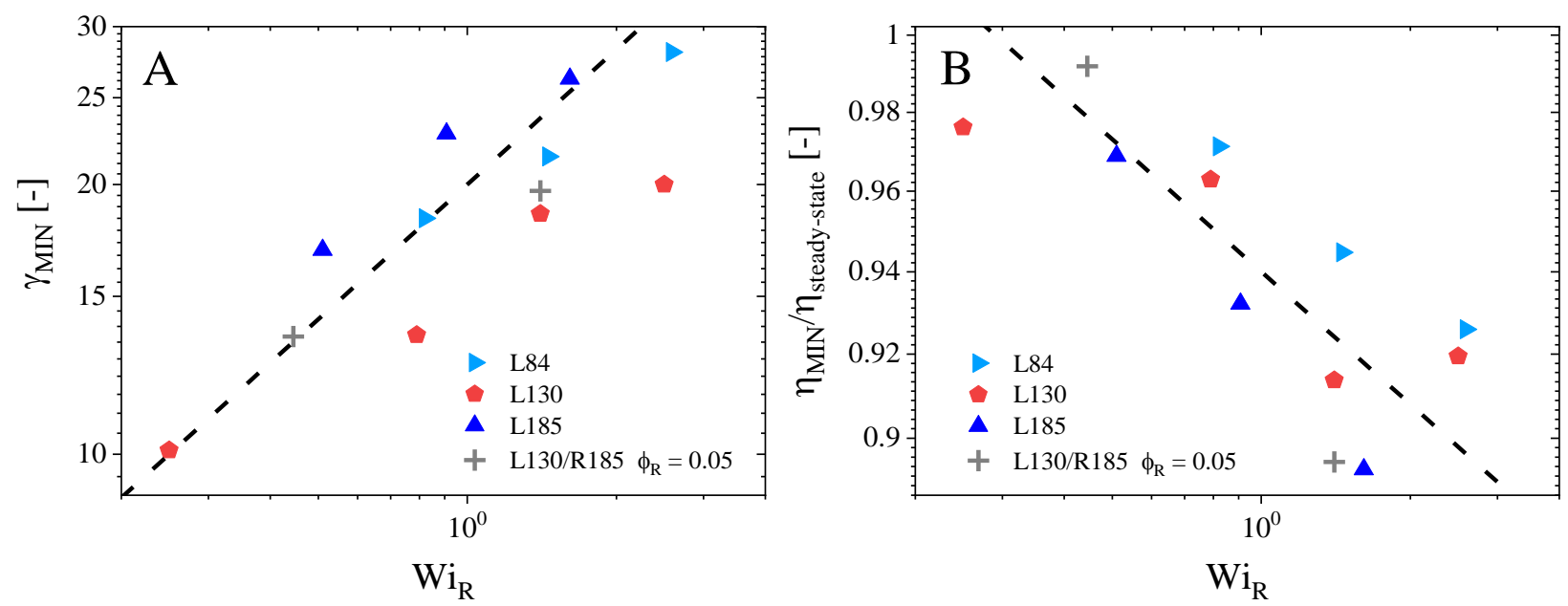

Figure 5. Analysis of the transient undershoot: Strain at minimum shear stress growth coefficient (A) and fractional undershoot (normalized), i.e., the ratio of minimum to steady-state shear stress growth coefficient values (B), as functions of the Rouse Weissenberg number. Error bars are within the symbol size. Dashed lines are a guide for the eye. 

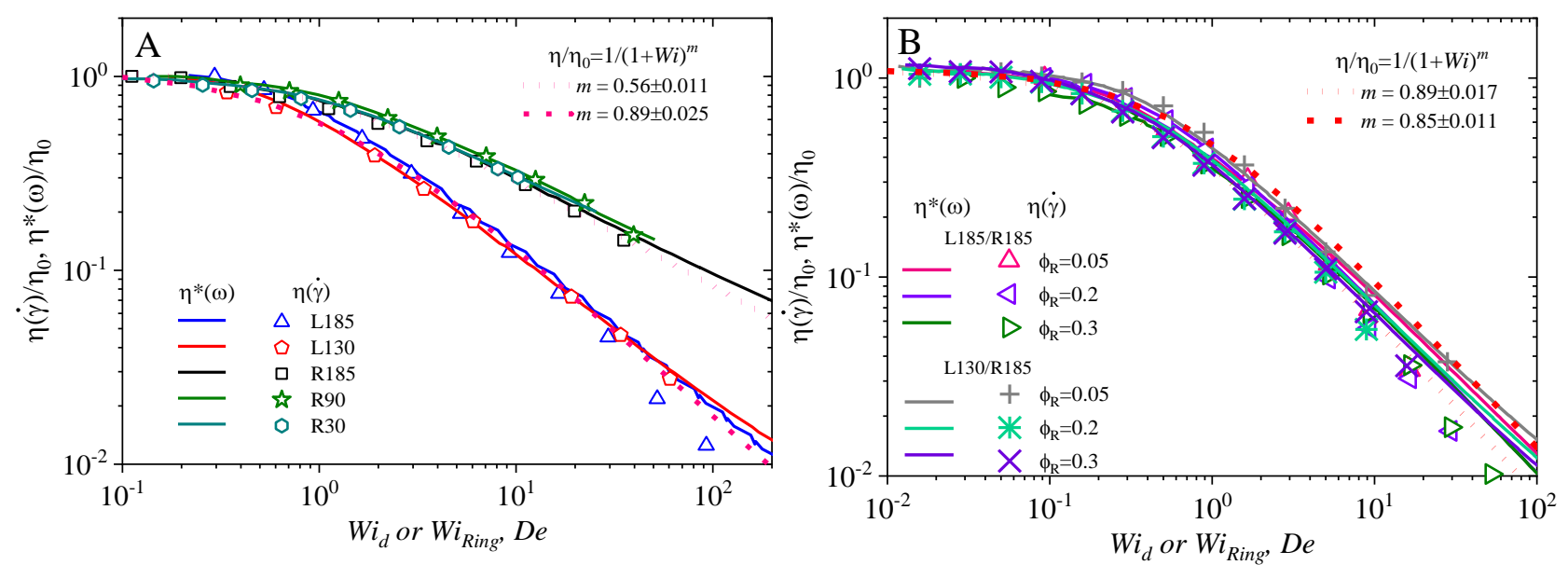

Figure 6. Steady-shear (symbols) normalized by the zero-shear rate viscosity as a function of the shear rate normalized by the terminal relaxation time (terminal relaxation Weissenberg number Wid or WiRing) and complex viscosity (lines) normalized by the zero-shear rate viscosity as a function the normalized frequency (Deborah number De) for the pure components (A) and the blends (B). The dashed and dotted lines in panel A are fits with the Carreau-Yasuda model for the linear and ring polymers, respectively. The dashed and dotted lines in panel B are fits with the Carreau-Yasuda model for the samples with small and large fractions of rings, respectively. The shear thinning exponents are reported in the Figure.

A measure of the degree of deformability of polymers under shear is the fractional overshoot, i.e., the ratio of the maximum value of the shear stress growth coefficient to its steady-state values. The fractional overshoot is plotted as a function of the Rouse-Weissenberg number WiR in Fig.7, where we can clearly distinguish two groups: the pure rings collapse into one group and follow a power-law dependence $\eta_{M A X} /\left.\eta_{\text {steady-state }}\right|_{\text {rings }} \sim W i_{R}{ }^{1 / 7}$, while all other samples (linear polymers and blends) appear to follow a distinctly different scaling behavior $\eta_{M A X} /\left.\eta_{\text {steady-state }}\right|_{\text {linear,blends }} \sim W i_{R}{ }^{1 / 4}$ with larger fractional overshoot values. The latter dependence has been reported for many different entangled linear polymers $[3,12,34,56]$. We note also that theoretical predictions based on the tube model with appropriate adjustments to account for segmental orientation and stretching do capture the occurrence of overshoot $[34,58,59]$. A recent tube-based model, which takes into account the concept of gripping force between topological constraints at shear rates exceeding the reciprocal Rouse time, also predicts overshoot in stress growth function [60,61]. However, the extent of fractional overshoot and its experimentally observed shear rate dependence were not captured quantitatively. The experimental findings of Fig. 7 suggest that the degree of deformability is larger in the pure melts of linear polymers and linear/ring blends compared to the pure rings. Moreover, a closer look at the data indicate that ring-linear blends exhibit slightly larger fractional overshoot 
compared to their respective pure linear component (see inset panel in Fig.7). Actually, the dependence of overshoot on blend composition appears to be non-monotonic; it is tempting to recall the non-monotonic dependence of zero-shear viscosity on composition $[4,5,6]$, but the data are too limited to draw definite conclusions. Hence, the threading of rings by linear chains should play a role in the enhanced deformation, and it also appears that the asymmetric blends (here with shorter linear component) could be slightly less effective in this respect. However this needs further investigation.

The strain, $\gamma_{\text {MAX, at }}$ at the overshoot (maximum) is plotted against Wir in Fig.8. A tendency of all data to follow a generic trend, albeit with some scatter, is observed. Interestingly, the high-WiR scaling $\gamma_{M A X} \sim W i_{R}{ }^{1 / 3}$ has been reported for different entangled linear polymers [34,56] and predicted by the tube-based model accounting for the gripping force [59,60]. The limited data of linear and ringlinear blends at $\mathrm{Wi}_{\mathrm{R}}<1$ virtually collapse at the value of 2.3 , the Doi-Edwards prediction for the segmental alignment in flow [26], while the rings do not seem to reach a plateau but rather exhibit a weak dependence on WiR (which is slightly stronger for R30). For WiR > 1, there is stretching without clearly distinguishable signatures between ring and linear polymers or their blends. Along these lines, the Hencky strain at the maximum value of the measured elongational stress growth coefficient is shown as a function of $\mathrm{Wi}_{\mathrm{R}}$ in the inset of Fig. 8 for pure ring and linear components and their blends, as indicated in the caption (see also relevant discussion below). Over the examined regime of $\mathrm{Wi}_{\mathrm{R}}<$ 1 , the elongational deformation is larger than the shear one, especially for the R185 sample exhibiting the highest fractional deformation [8,9]. The peculiar extensional response of R185 is reported elsewhere [9]. 


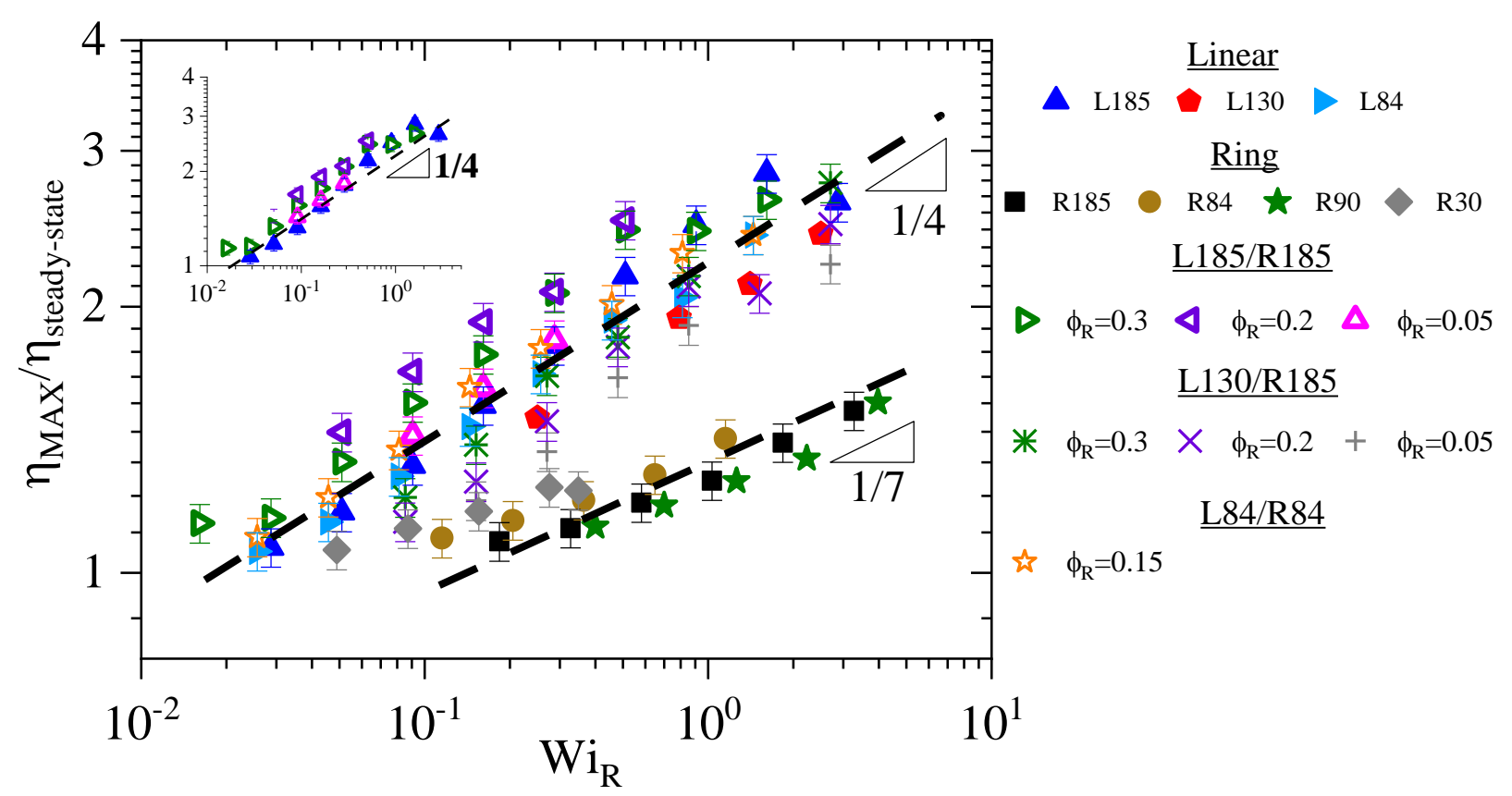

Figure 7. The maximum overshoot in the shear stress growth coefficient normalized by its steadystate value as a function of the Rouse-Weissenberg number. The black dashed lines with slopes 1/4, and 1/7 indicate the dependence observed in earlier analysis of entangled linear and marginally entangled ring polymers, respectively [7,31]. Data for the 84k linear (L84) and ring (R84) polymers, as well as their blend L84/R84 are taken from literature [3]. The inset presents the maximum shear stress growth coefficient normalized by its steady-state value for L185 and the symmetric L185/R185 blends. Symbols are listed in the legend and refer to Table 1. Error bars refer to the errors in determining $\eta_{M A X}$ and $\eta_{\text {steady-state }}$. When not clearly visible, the error bars are within the symbol size. 

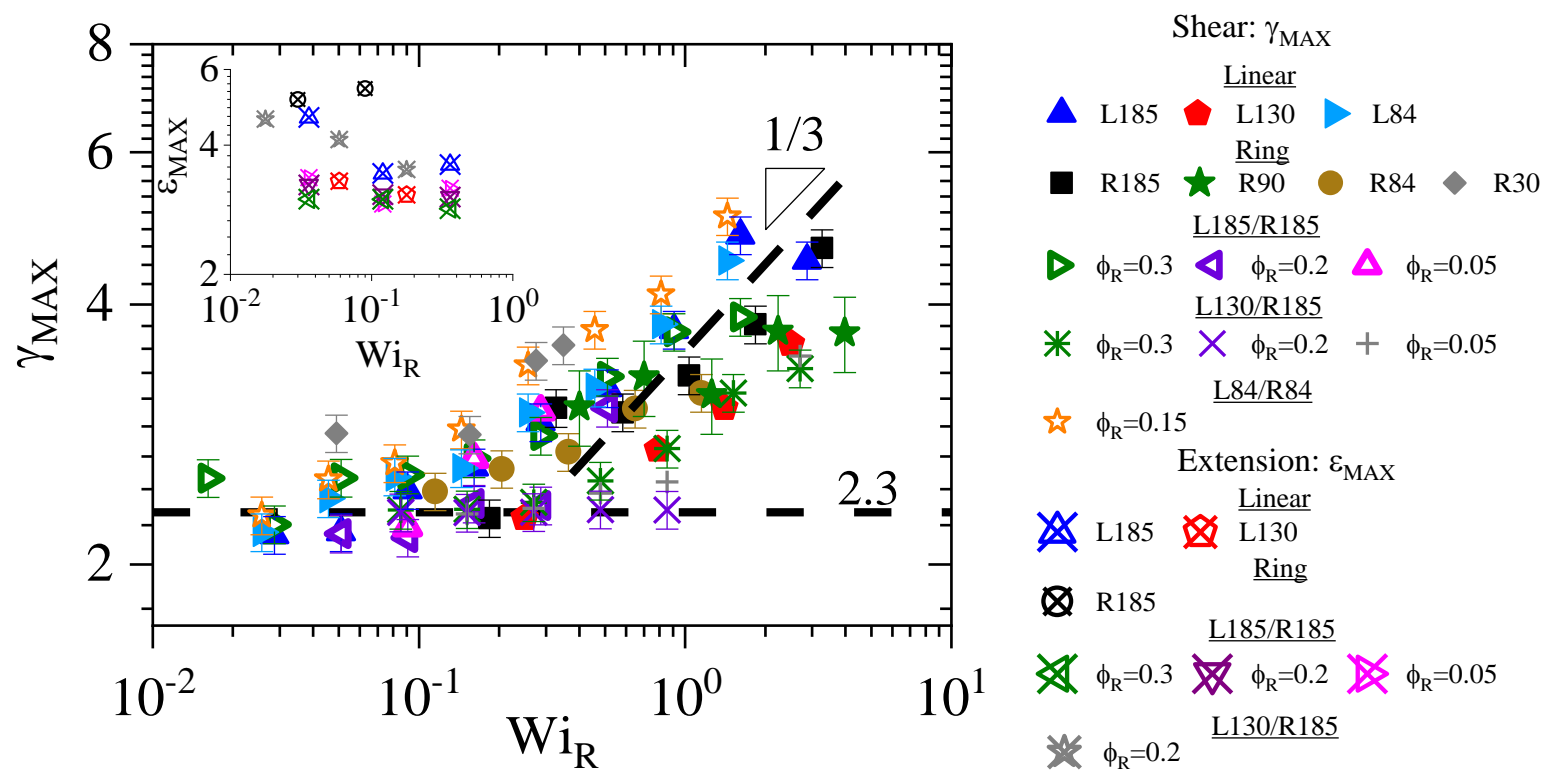

Figure 8 . Shear strain, calculated as the product of time and shear rate $(\dot{\gamma})$, at the maximum overshoot of the shear stress growth coefficient, $\dot{\gamma}$ as a function of the Rouse-Weissenberg number WiR for pure components and blends. Symbols are reported in the legend and refer to Table 1. The horizontal dashed line at $\gamma_{\operatorname{MAX}}=2.3$ represents the Doi-Edwards prediction for monodisperse linear polymers [26], whereas the dashed line with slope $1 / 3$ indicates the dependence predicted by the analysis of entangled linear and marginally entangled ring polymers, respectively [7,31]. The inset displays the Hencky strain at the maximum elongational stress growth coefficient as a function of $\mathrm{Wi}_{\mathrm{R}}$ (product of the Hencky strain and rate $\dot{\varepsilon}$ ). Data for the 84k linear (L84) and ring (R84) polymers, as well as their blend L84/R84 are taken from the literature [3]. Error bars represent the error in determining

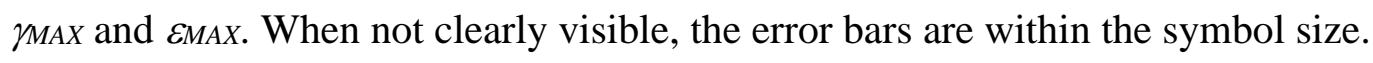

\section{Steady-state normal stresses.}

The measurements of the normal stress differences, especially $\mathrm{N}_{2}$, are much more challenging $[12,32]$. In Fig. 9 we present the apparent first normal stress difference obtained as described in section 2.1 above. Note that the transient data are not taken into consideration because they are affected by axial compliance as discussed in detail in Ref. [35]. Hence, we focus on the steady-state values. Additional experimental data, as well as reliability and reproducibility tests are presented in the SI (Figs.S14S20). The insets of Fig. 9 demonstrate the significant result that the steady-state has been reached. The cut-off in the overshoot data reported in Panel C at $\dot{\gamma}=56.2 \mathrm{~s}^{-1}$ is due to the upper limit of the normal force transducer, reached during the particular experiment with a $4 \mathrm{~mm} \mathrm{CPP}$. We note that, 
despite the reproducibility of the data, there are non-negligible fluctuations rendering uncertainties in the result. In this context, we note that the irregular oscillations observed in the signals of Fig. 9 do not reflect material properties but rather experimental issues. Actually, we have observed the lower quality of the apparent first normal stress difference for the smaller diameter of the plate at a given shear rate. This is due to a trade-off associated with (i) the need to obtain a signal with value below the upper limit of the axial transducer and above the resolution threshold and (ii) avoiding flow instabilities including edge fracture.

By using our method outlined in section 2.1, we extracted the true experimental first and second normal stress differences, which are plotted as functions of the Weissenberg number in Fig.10.The selected time for $\mathrm{Wi}$ is the terminal time assigned to the linear polymers, blends or rings (Table 2). In other words, we do not account for the slow mode of the rings, as discussed in Refs. $[4,9,11]$. Further, we observe that $N_{2}<0$ and $\left|N_{2}\right|<N_{1}$ always, which is consistent with results from recent non-equilibrium molecular dynamics simulations $[16,62]$ and will be further elaborated below. Given the inevitable experimental uncertainties, despite the extra care taken during the measurements, and relatively small range of accessible rates (Table 3 ) it is hard to draw definite conclusions about the dependence of the two normal stress differences on Wi. Their differences, if any, are small, as also confirmed by the recent molecular dynamics simulations with polyethylene, which for the larger investigated chains $\left(\mathrm{C}_{400} \mathrm{H}_{800}\right)$ suggest a power-law dependence of $N_{1}$ and $\left|N_{2}\right|$ with average powerlaw exponent of $0.6[16,62]$. Note that the reported respective exponents for linear chains, based on results of non-equilibrium molecular dynamics simulations for linear polymer melts (of chain length up to $\mathrm{N}=400$ ) are higher, 0.67 and 0.82 [63]. 

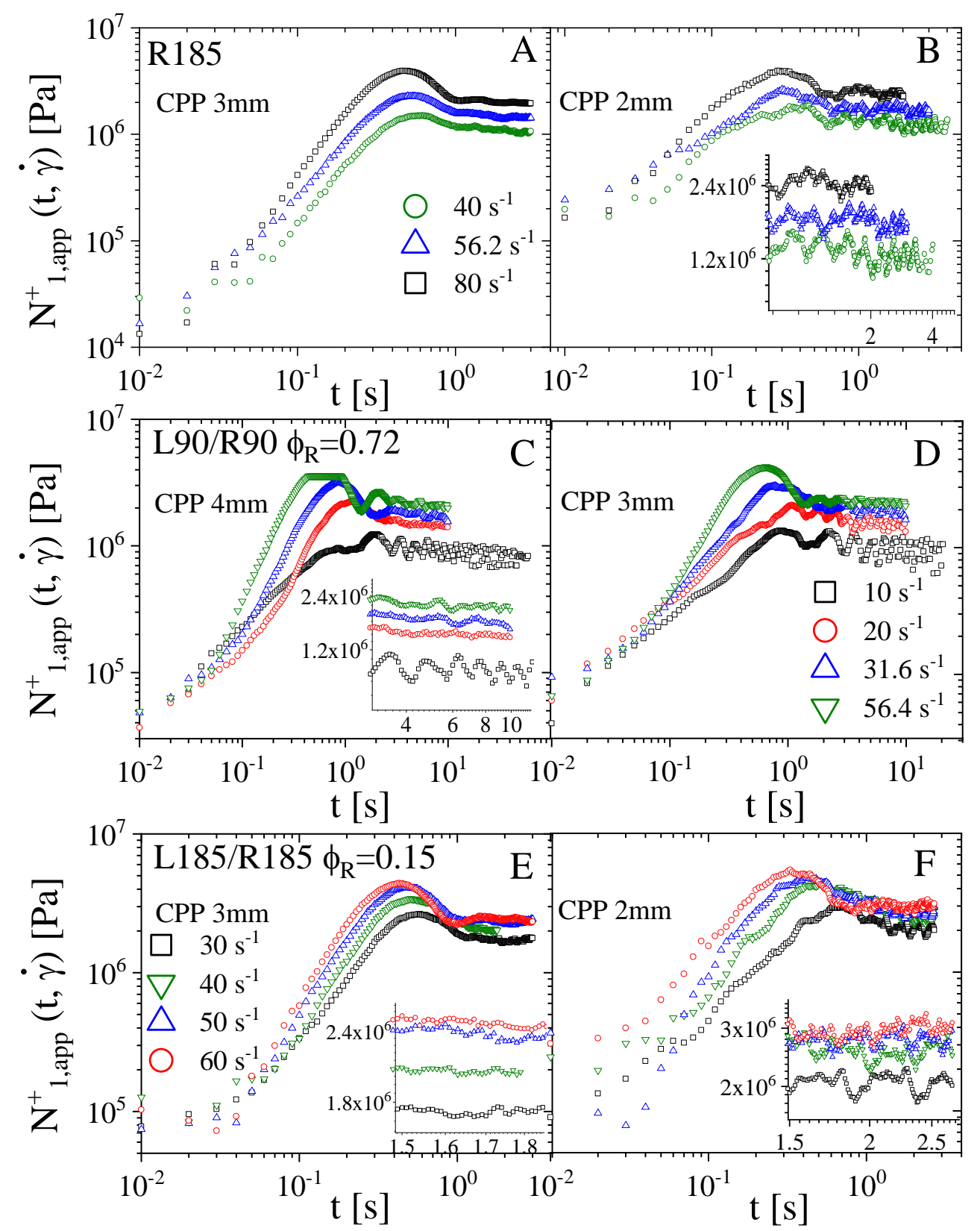

Figure 9. Start-up of shear rate in terms of apparent first normal stress difference, $\mathrm{N}_{1 \text {,app }}$, as a function of time at various shear rates for: (i) ring R185 with A) CPP $3 \mathrm{~mm}$ and B) CPP 2mm; (ii) blend L90/R90 at $\phi_{\mathrm{R}}=0.72$ with C) CPP 4mm and D) CPP 3mm; blend L185/R185 at $\phi_{\mathrm{R}}=0.15$ blend with E) CPP 3mm and F) CPP 2mm. Results for R185 and L90/R90 at $\phi_{\mathrm{R}}=0.72$ blend refer to $150{ }^{\circ} \mathrm{C}$, whereas for L185/R185 at $\phi_{\mathrm{R}}=0.15$ to $160^{\circ} \mathrm{C}$. Insets in Panels B, C, E, and F show a magnification of the steady-state region. Same legends hold for panels (A,B), (C,D) and (E,F). Data for the R30 samples are showed in Figure S14 of the SI. 


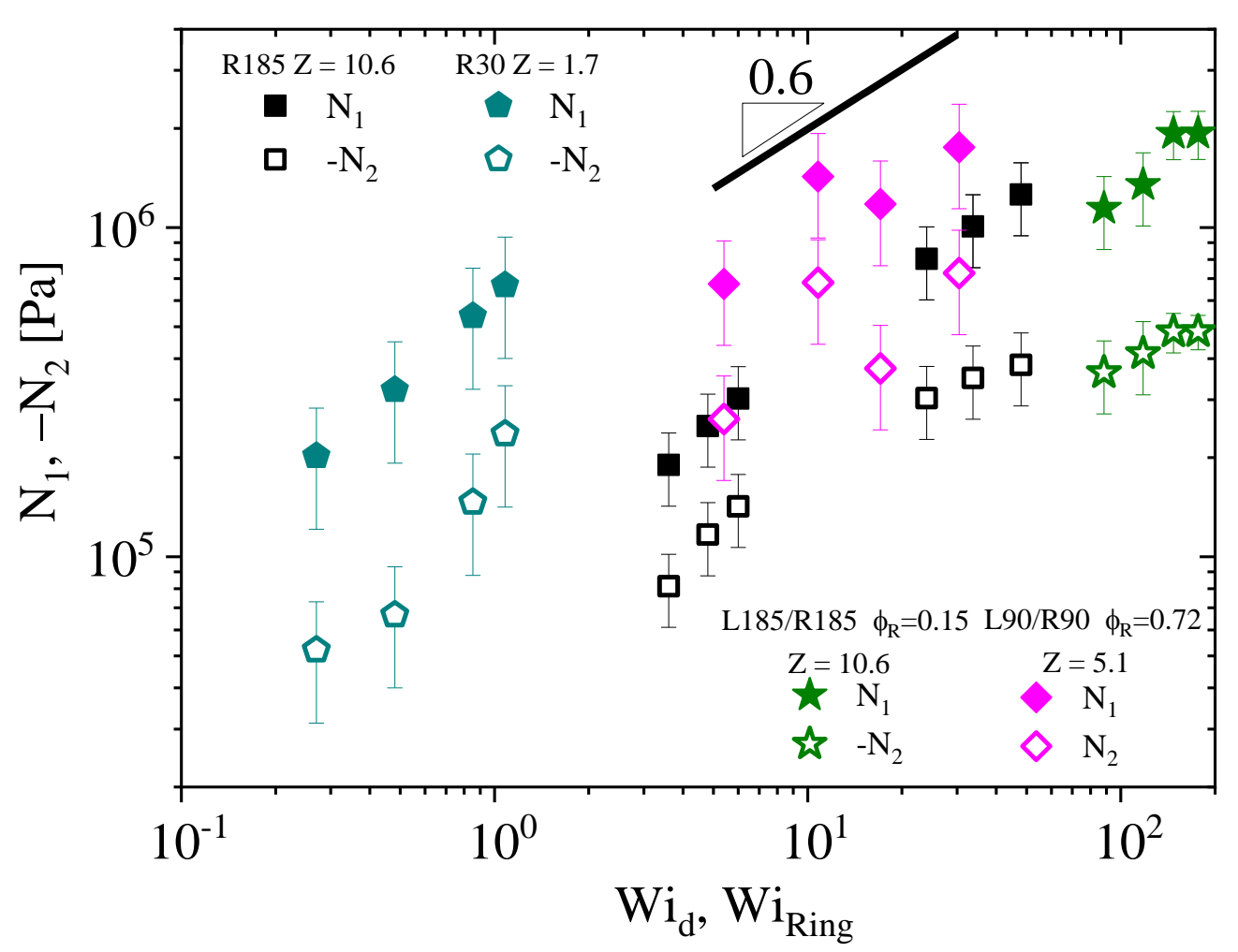

Figure 10. Steady-state values of the first $\mathrm{N}_{1}$ (solid symbols) and (minus) second $-\mathrm{N}_{2}$ (open symbols) normal stress differences as a function of the terminal Weissenberg number (based on the terminal time for linear or blend $\mathrm{Wi}_{\mathrm{d}}$ or the extracted ring time $\mathrm{Wi}_{\mathrm{Ring}}$ ) for ring $\mathrm{R} 185$, blend $\mathrm{L} 90 / \mathrm{R} 90$ at $\phi_{\mathrm{R}}=$ 0.72 and blend L185/R185 at $\phi_{\mathrm{R}}=0.15$. The slope represents non-equilibrium molecular dynamic simulations results for ring polyethylenes [63]. Error bars are calculated by averaging out at least 20 data points in the steady-state.

Fig.11 depicts the Wi-dependence of the ratio $-\mathrm{N}_{2} / \mathrm{N}_{1}$ for the measured polymers. For completeness, we add two entangled linear polystyrene solutions (in oligomers) from Ref. [12]. The value of $-\mathrm{N}_{2} / \mathrm{N}_{1}$ ratio is higher for pure rings and ring-linear blends, when compared to linear polymer solutions [12]. Further, a comparison based on the number of entanglements at the same Weissenberg number is more revealing. Starting from the linear polymer solutions, there seems to be a decrease of the ratio as $\mathrm{Z}$ increases from 6 to 29. This was attributed to the fact that samples with broader relaxation spectrum have more relaxed (faster) modes, which are not oriented and therefore have lower $\mathrm{N}_{1}$ and contribute to larger $-\mathrm{N}_{2} / \mathrm{N}_{1}$ ratio [12]. Consequently, when the spectrum approaches single-mode limit in the terminal regime, which is more evident for larger $\mathrm{Z}$, this ratio decreases. This argument may also explain the hint of larger $-\mathrm{N}_{2} / \mathrm{N}_{1}$ ratio for rings. 
Taking this as a basis, the limited high-Wi data of the $185 \mathrm{k}$ blend with only $15 \mathrm{wt} . \%$ of rings and $\mathrm{Z}$ $=10.6$ appear to conform to this picture. We already know that threading of rings by linear polymers strongly influences the linear and nonlinear extensional properties of the blends [4,8]. More striking are the results with the $90 \mathrm{k}$ blend with $\mathrm{Z}=5.1$ and, importantly, the pure $185 \mathrm{k}$ ring with $\mathrm{Z}=10.6$ : the $-\mathrm{N}_{2} / \mathrm{N}_{1}$ ratio is clearly higher and almost constant at about 0.4 over a range of Wi covering almost 1.5 decades. This strongly suggests that ring-linear threading is very important in nonlinear shear response. The added rings are not a simple but a topological 'solvent', as discussed recently [4]. Moreover, the presence of even small fraction of rings (0.15) clearly affects the ratio $-\mathrm{N}_{2} / \mathrm{N}_{1}$ (see data in Fig.11 at large values of Weissenberg number). The experimental evidence is unfortunately limited and scattered due to the difficulty in obtaining appropriately purified ring polymers in sufficient amounts.

We now review recent results from atomistic molecular dynamics simulations with two different polymers, polyethylene [61] and poly(ethylene oxide) [16]. In the former work, the authors investigated $\mathrm{C}_{78} \mathrm{H}_{156}$ and $\mathrm{C}_{400} \mathrm{H}_{800}$ (the latter corresponding to $\mathrm{Z} \sim 4.5$ ) rings and their linear counterparts and found that the ratio $-\mathrm{N}_{2} / \mathrm{N}_{1}$ is always larger for the rings (double) and depends on the macromolecular size. The respective ring/linear $-\mathrm{N}_{2} / \mathrm{N}_{1}$ ratios were $0.4 / 0.2$ and $0.2 / 0.1$ for $\mathrm{C}_{78} \mathrm{H}_{156}$ and $\mathrm{C}_{400} \mathrm{H}_{800}$, respectively. In the latter work, polymers with $\mathrm{Z} \sim 0.5,1$ and 2.5 and revealed that in all cases the ratio $-\mathrm{N}_{2} / \mathrm{N}_{1}$ was higher for rings, with the difference decreasing with $\mathrm{Z}$ and exhibiting weak shear rate dependence. 


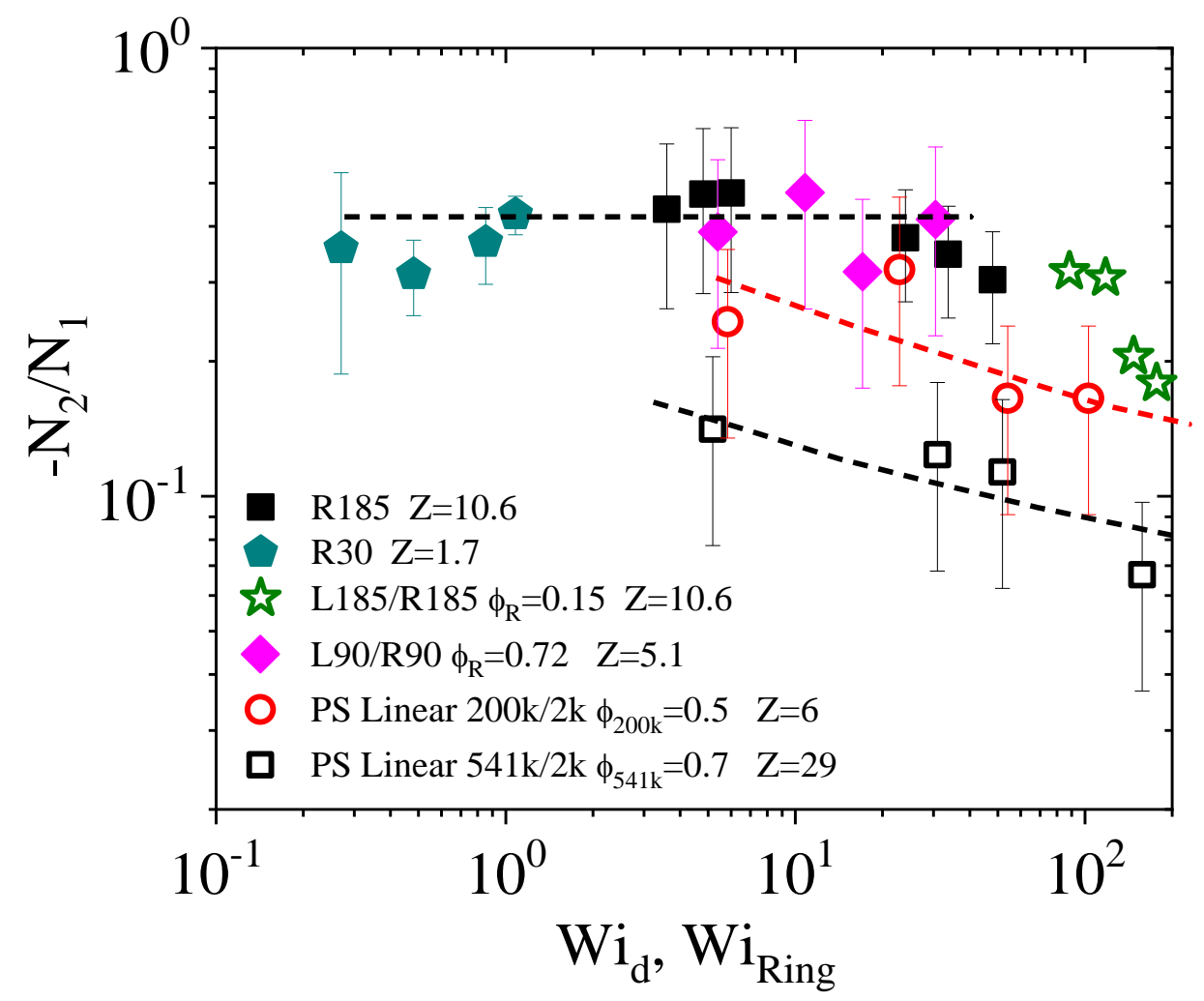

Figure 11. Ratio of experimental steady-state (minus) second $-\mathrm{N}_{2}$ and first $\mathrm{N}_{1}$ normal stress differences as a function of the terminal Weissenberg number, $\mathrm{Wi}_{\mathrm{d}}$ or $\mathrm{Wi}_{\text {Ring, }}$, for $\mathrm{R} 185$ (solid squares), R30 (dark cyan pentagons), L90/R90 at $\phi_{\mathrm{R}}=0.72$ (solid diamonds) and L185/R185 at $\phi_{\mathrm{R}}=0.15$ (open stars). For comparison, respective data from two PS solutions (long linear polymers diluted in oligomers) taken from the literature (open circles and squares) [12] are shown. Lines are drawn to guide the eye. Error bars are calculated by averaging at least 20 data points in the steady-state.

We now compare the experimental data with recent predictions of the shear slit model [11]. According to this model, the sheared ring is compressed in the velocity gradient direction into a slit of the shear blob size, giving rise to negative $N_{2}$ which is controlled by the number of monomers in a shear blob $g_{s}$ (see inset of Figure 12). At the same time, the ring is stretched in the flow direction, with $\sigma_{x x}>\sigma_{z z}$, and $\sigma_{x x}-\sigma_{z z}=N_{l}+N_{2}$ being controlled by the number of monomers in a tension blob gt. This yields the following predictions:

$$
N_{1}(\dot{\gamma})+N_{2}(\dot{\gamma})=\frac{k T \rho}{\mathrm{g}_{\mathrm{t}}(\dot{\gamma})}
$$


$N_{2}(\dot{\gamma})=-\frac{k T \rho}{\mathrm{g}_{s}(\dot{\gamma})}$

where $\rho$ is the number density of monomers. The stronger tension in the flow direction (smaller tension blob compared to shear blob, see also inset of Fig.12 and Ref.[11]) means that $N_{l}>-N_{2}$. Therefore, Eq. (6) suggests that $N_{1}(\dot{\gamma}) \sim \mathrm{g}_{\mathrm{t}}(\gamma)^{-1}$. The slit model predicts [11] $\mathrm{g}_{s}(\dot{\gamma}) \sim \dot{\gamma}^{-3 / 7}$ for $\mathrm{Wi}_{\mathrm{d}}>1$, hence $-N_{2} \sim \dot{\gamma}^{3 / 7}$. On the other hand, $\mathrm{g}_{t}(\dot{\gamma}) \sim \dot{\gamma}^{-x}$ with the exponent $\mathrm{x}$ decreasing from $12 / 7$ to $8 / 7$ beyond a crossover shear rate value of $Z^{7 / 4} / \tau_{e}$. This translates in respective power-law exponents for first normal stress difference $N_{l}$ of $12 / 7$ and $8 / 7$.

In Figure 12 we plot the number of blobs per chain as function of the terminal Weissenberg number by scaling the experimental normal stress data with $k T \rho_{N}=\frac{N_{A} \rho_{\text {polystyrene }}}{M_{w}} k T$, where $\mathrm{N}_{\mathrm{A}}$ is the Avogadro number and $\rho_{N}$ is the number density of number density of chains. In general, in this representation one may appreciate that the number of blobs per chain increases with $W i_{\text {Ring }}$, or alternatively their size decreases. We note that $-N_{2}$ has a slope 3/7 at higher Wiring values, but for Wiking $\sim 1$ (which corresponds roughly to one shear blob per chain) there seems to be a crossover toward a steeper, linear dependence. For $W i_{\text {Ring }} \leq 1$ the $N_{l}$ data exhibit a similar linear dependence, which however becomes weaker at larger values of $W i_{\text {Ring }}>1$, departing from the predictions; the data seem to comply with the slope of about 0.6 , found in recent simulations studies, as discussed above. Whereas it seems that at lower rates the dependence is slightly stronger and a crossover may take place similarly to the $-N_{2}$ case, this cannot be discerned given the experimental uncertainties, so we refrain from further discussing. 


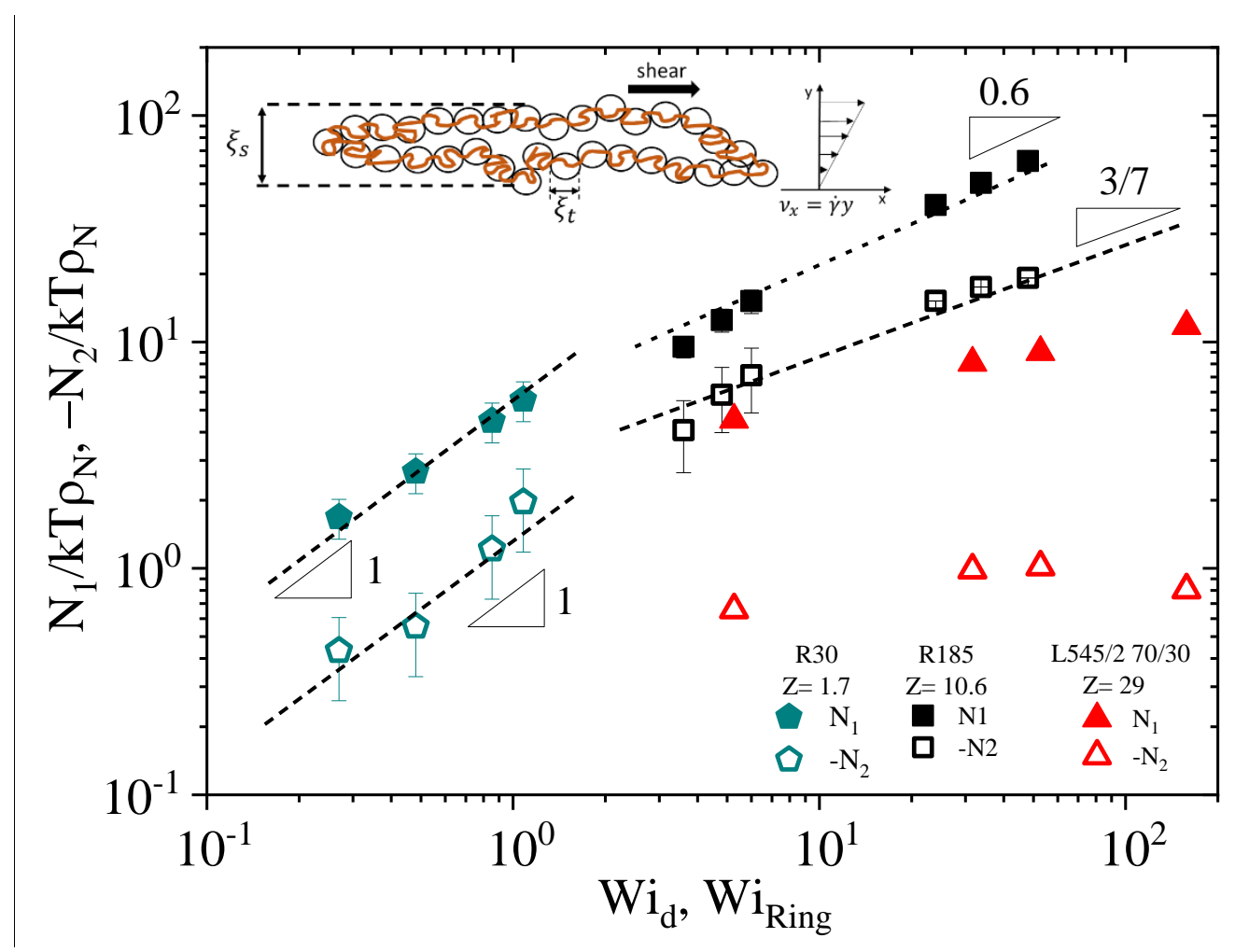

Figure 12. Normalized steady-state values of the first and second normal stress differences by the thermal energy $\mathrm{kT}$ and the number density of chains $\rho_{\mathrm{N}}$, i.e., number of blobs per chain, as functions of the terminal Weissenberg number Wid, or WiRing. Samples R30, R185 and a linear polymer solution from Ref. [12] are reported as dark cyan pentagons, black squares and red triangles, respectively. Black dashed lines depict the $3 / 7$ and 1 slope for the second normal stress difference data, and the 0.6 and 1 slope for the first normal stress difference (see discussion in the text), respectively.

The discussion and proposed interpretation of the rheometric data presented and analysed above can be summarized graphically in Fig.13 (top), where cartoons of entangled linear polymers, entangled ring polymers and their blends deformed in a shear field at large Weissenberg number are depicted. The message is the illustrated different deformed conformations, with stretched linear polymers forming long filaments, rings deformed in shear slits (inset of Fig.12), possibly with some interlocking at very large rates, and blends characterized by deformation of the threaded rings and some unthreading. Undoubtedly, a quantitative interpretation calls for further modeling and simulations, let alone experiments. However, the key point is the consistency of the data and the possible role of the recently revealed mechanisms associated with deformed ring ( e.g., threadingunthreading transition, interlocking, weak shear thinning $[3,4,8-11,17])$ in the transient shear and steady normal stress responses. The above scaling model [11] represents a promising framework to understand the molecular origins of $N_{1}$ and $N_{2}$. 

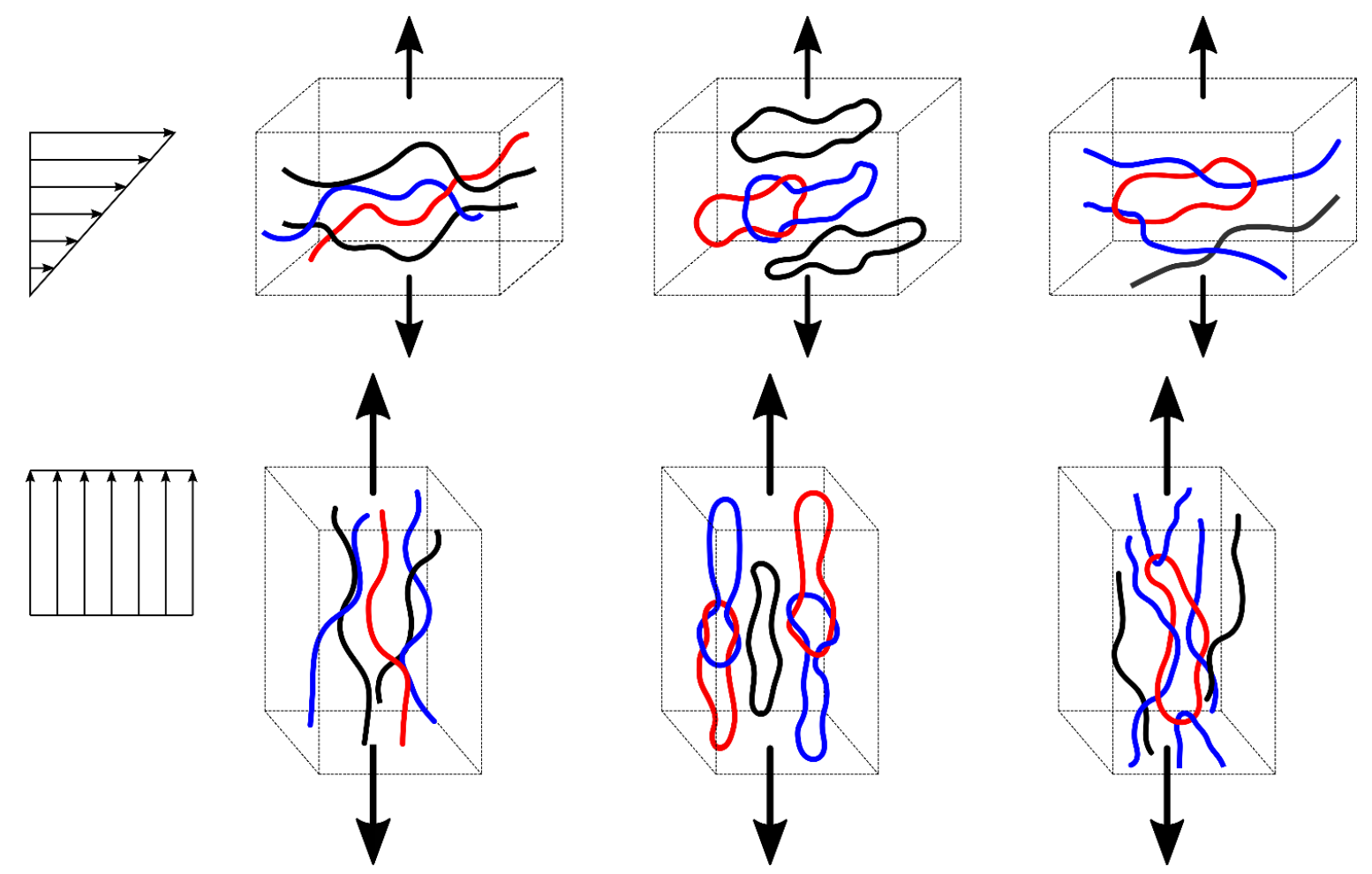

Figure 13. Schematic illustrations of strongly deformed (at a Rouse-Weissenberg number above 1) entangled linear polymers (left), entangled ring polymers (middle) and ring-linear blends (right). Different colors (black and grey in print) are chosen for clarity of presentation (to identify individual polymers). Two deformation fields are depicted, shear (top) and uniaxial extension (bottom).

\section{Comparison of shear and elongational response: stress growth coefficients and stresses.}

It is interesting to compare the shear and extensional data (see also Fig.S22 of the SI). We do so in Fig. 14 for the blends and pure components, where their viscosities are plotted as function of the Rouse-Weissenberg number, Wir. As a background, we note that the low-Wir Newtonian Trouton ratio of 3 and high-Wir thinning slope of linear polymers are validated, whereas rings exhibit qualitatively different behaviour reflected in the low-WiR thickening due to ring inter-locking $[13,38]$, so the validity of low-WiR Trouton ratio remains unclear. Note that in this representation, the elongational data of pure linear and ring components L185 and R185 do not collapse in the regime of large $\mathrm{Wi}_{\mathrm{R}}$, since they do so at the same elongational rates as discussed in Ref. [9]. Here, we focus on the blends for which we have sufficient experimental evidence. Based on earlier works $[8,9,17]$, the elongation of linear and ring polymers as well as their blends is graphically illustrated in Fig.13 (bottom). We also recall that in the available Wir measured (lower for elongation), the achieved deformation is stronger in elongation compared to shear (Fig.8 inset). Referring to Fig.14, given that 
the steady-state values of the elongational stress growth coefficient were not probed because of the sudden drop of its value after the overshoot due to the threading-unthreading transition [6], the elongational stress growth coefficient at the maximum are reported. These values are above the respective Trouton values, as expected (since they are not the steady-state elongational viscosity values). Further, the thinning slopes of the blends follow those of the linear component, i.e., -0.82 and -0.5 for shear and elongation, respectively. From these observations the need for further investigations of the conformational changes of threaded ring-linear blends in shear and extension by means of simulations and targeted neutron scattering experiments is evident.

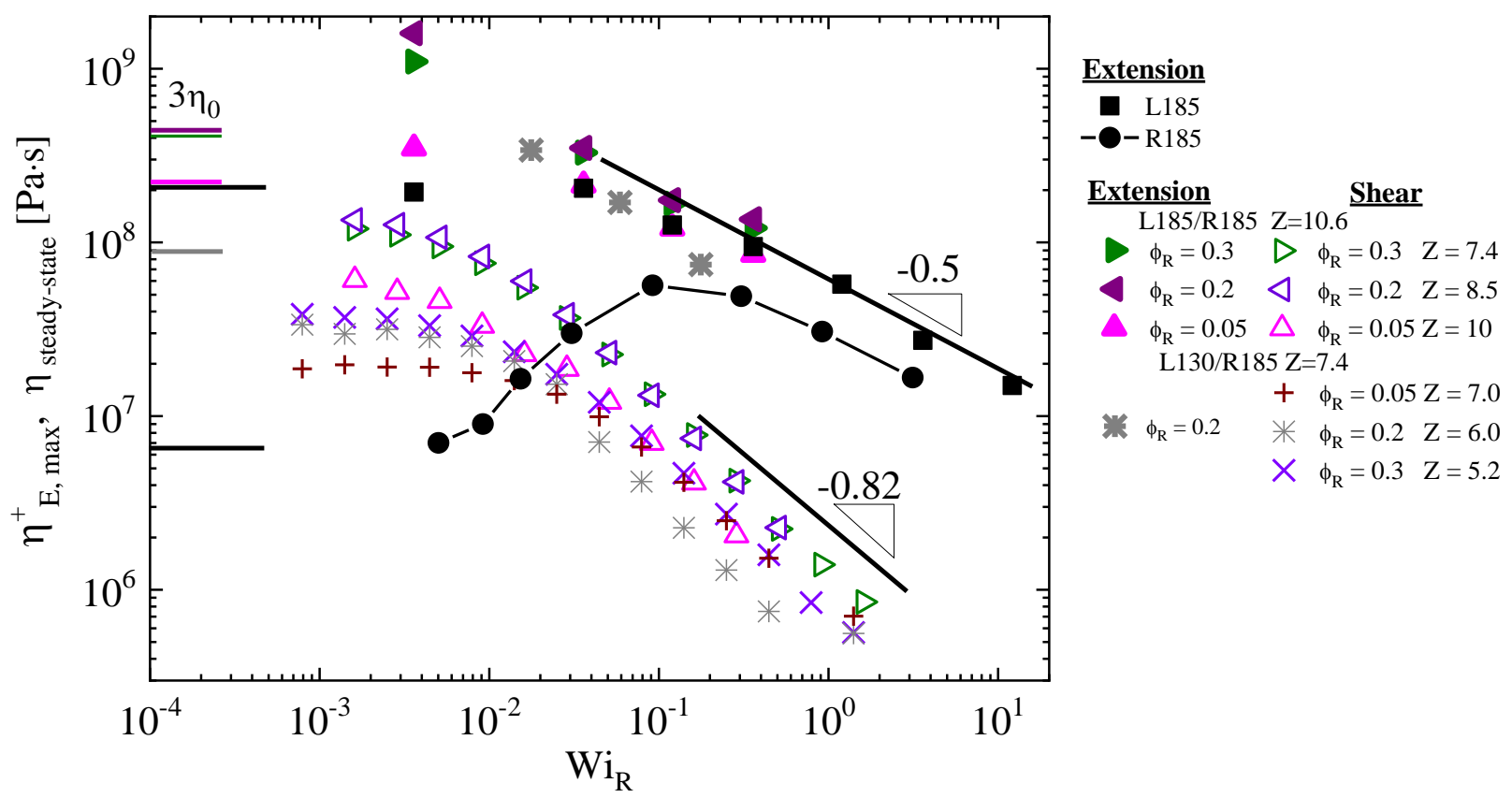

Figure 14. Maximum elongational stress growth coefficients and steady-state shear viscosites as a function of the Rouse-Weissenberg number for ring-linear blends at $130{ }^{\circ} \mathrm{C}$. Extensional data for L185/R185 at $\phi_{\mathrm{R}}=0.3$ are taken from Ref. [8]. Details are reported in the legend. The solid lines to the left denote the values of $3 \eta_{0}$ for each sample (larger lines correspond to the pure L185 and R185 components). The slopes -0.5 and -0.82 describe the thinning power-law slopes in for linear polymers in uniaxial extension and shear, respectively $[3,9,11,65]$. Error bars calculated by averaging out at least 20 data points in the steady-state are within the symbol size.

We now compare in Fig. 15 the measured normal stresses in shear and elongation. Given the experimental difficulties identified above, only limited systematic data are presented. Moreover, for the same reasons, the data for the L185 sample in shear were obtained with one CPP geometry only,

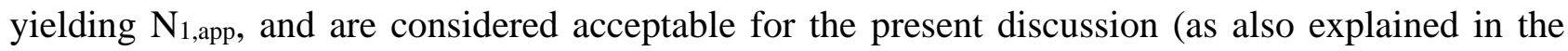
experimental section 2.1 above). This could be corroborated by the fact that the three sets of data, 
$\mathrm{N}_{1, \text { app }}$ for L185 and $\mathrm{N}_{1}$ and R185 and their blend at $\phi_{\mathrm{R}}=0.15$, nearly collapse. The first observation from Fig. 12 is that the elongational stress is much larger compared to the first normal stress difference in shear. This difference is further enhanced in pure rings compared to linear polymers (our data suggest respective values of 20 and 7 for the ratio $\left\langle\sigma_{\mathrm{zz}}-\sigma_{\mathrm{rr}}>/ \mathrm{N}_{1}\right.$ at the largest rates investigated). This is not surprising given that shearing motion involves both normal and shear stresses. Along these lines, it is interesting to note that the low-rate R185 ring data in elongation deviate from the established behaviour of linear polymers [60,61], according to which the elongational stress is proportional to stretch rate, not to its square. This conforms to the strong thickening of rings at low elongational rates due to interlocking [17]. Unfortunately, respective low-Wi normal stress data in shear rheometric experiments could not be obtained due to instrument resolution limitations. However, application of Laun's rule [67,68] suggests that the so-extracted elongational stress ring data follow the known low-shear-rate scaling with a power-law exponent of 2 (see Fig. S21 in SI). Finally, turning to the blends, the same observation holds, i.e., much larger elongational stress compared to normal stress in shear. The data with blend L185/R185 (with ring fraction not exceeding 0.3 ) at large rates of deformation follow the pure ring data (however the L185 data are apparent as discussed above). This is again consistent with recent findings pointing to the role of added rings in effectively reinforcing linear matrices $[4,8]$.

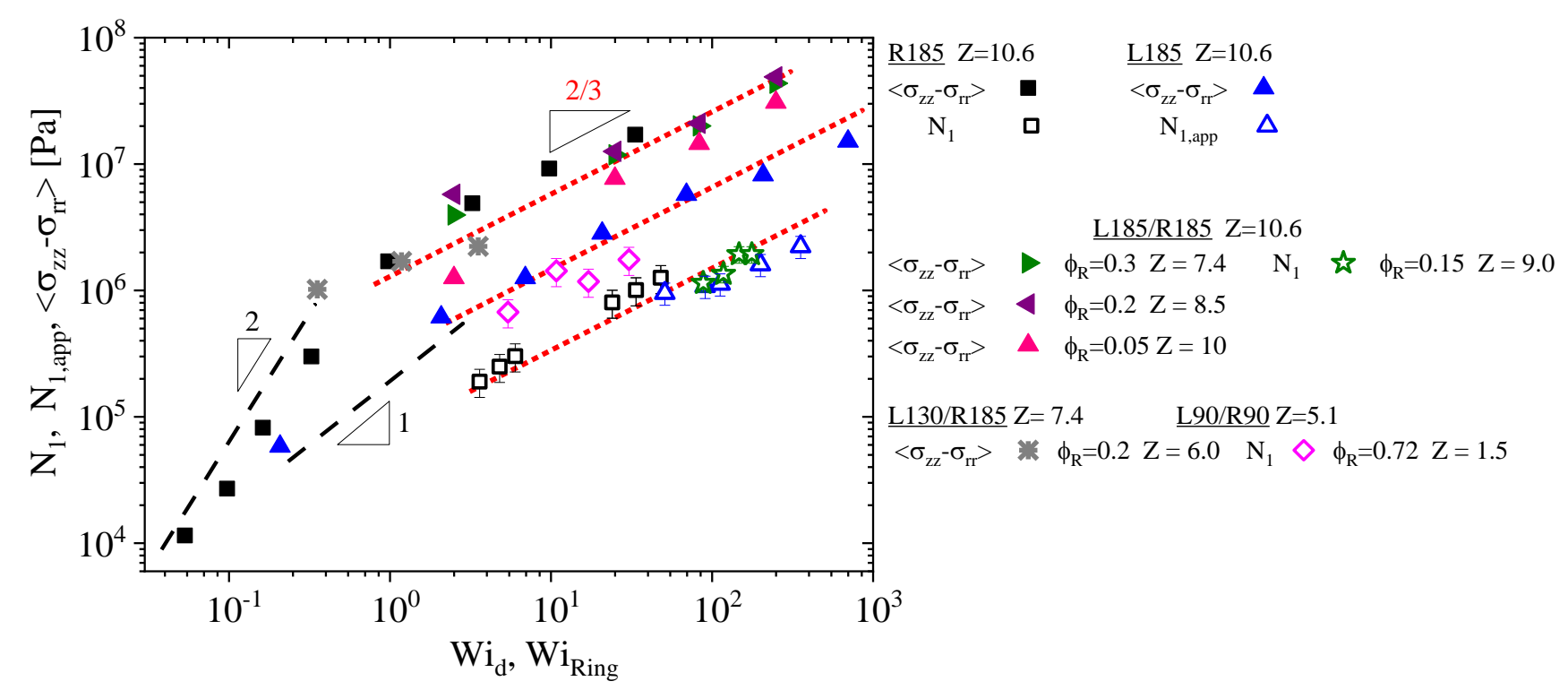

Figure 15. Representation of the measured first normal stress difference $\left(\mathrm{N}_{1}\right)$ or its apparent value $\left(\mathrm{N}_{1, \text { app }}\right.$, see text) in shear and extensional stress $\left.\left(<\sigma_{\mathrm{zz}}-\sigma_{\mathrm{rr}}\right\rangle\right)$ in elongation, as a function of the Weissenberg number based on terminal time ( $\tau_{\text {Ring }}$ for the rings, as noted above). Data (filled symbols 
for elongation, open for shear) are shown for blend L130/R185 at $\phi_{\mathrm{R}}=0.2$, linear L185, ring R185 [9] and various L185/R185 blends. The legend explains symbols and details. Lines represent limiting slopes or scaling predictions (see text). Error bars are calculated by averaging out at least 20 data points in the steady-state. When not clearly visible, they are within the symbol size.

\section{Conclusions and perspectives}

The systematic experimental study of the transient nonlinear rheology of purified entangled polystyrene rings and their blends with linear polystyrene chains has revealed several interesting new findings. The transient shear viscosity of rings barely exhibits undershoot in contrast to larger undershoot for linear chains at the same Weissenberg numbers. We expect that, if present and/or detectable, undershoot will appear at longer times at the same shear rate for rings, in comparison to their linear precursors. The shear thinning of the rings agrees with predictions of a shear slit model (with shear thinning exponent of -4/7). Further, the stress response of symmetric and asymmetric ring-linear (with smaller molar mass of linear component) blends with ring fraction up to $30 \%$ appears dominated by the linear component, as judged by the appearance of undershoot, scaling of the fractional overshoot and the strain at overshoot, as well as the shear thinning exponent. Both $N_{l}$ and $-N_{2}$ for rings and their blends, exhibit a weak shear-rate dependence. For $N_{l}$, the data for $W_{i R i n g}>1$ appear consistent with results from recent non-equilibrium molecular dynamics simulations which indicate a power-law dependence with exponent of about 0.6 , whereas for $W_{i R i n g}<1$ the seem to follow a linear dependence. Concerning $-N_{2}$, its rate dependence at large rates is consistent with predictions of the slit model (slope of 3/7), whereas there is a crossover to stronger dependence (slope of about 1) at lower rates corresponding to $W_{i R i n g} \sim 1$ and about one shear blob per chain. The ratio $-\mathrm{N}_{2} / \mathrm{N}_{1}$ for rings (of about 0.4 ) exceeds that of linear polymer solutions with the same number of entanglements by about factor of two. The larger $-\mathrm{N}_{2} / \mathrm{N}_{1}$ ratio is result is supported by the slit model and recent nonequilibrium molecular simulations with different polymers.

Comparison of viscosities and normal stresses in shear and elongation supports the different deformation mechanisms of ring and linear polymers (with similar final shape of extended chains and larger tension in elongation). The ratio $\left\langle\sigma_{\mathrm{zz}}-\sigma_{\mathrm{rr}}\right\rangle / \mathrm{N}_{1}$ is always much larger than 1 as expected, larger and rings and ring-linear blends compared to linear polymers, and increases with the rate of (shear or extensional) deformation. Finally, the ring response at low rates exhibits a much stronger dependence 
on stretch rate compared to linear polymers, with double power-law exponent. This evidence consistently conforms to the unique low-rate thickening of rings.

Overall, the addition of ring polymers has significant rheological consequences due to topological threading. Available data indicate that at relatively low fractions of rings, their blends with linear polymers exhibit features of both components: (i) signatures of linear in the rubbery plateau, transient shear overshoot and undershoot (although there is a need to clarify the possible role of molar mass asymmetry, here having considered the case of lower molar mass of linear component), the Widependence of fractional overshoot; and (ii) signatures of ring in the elongational stress (following rings) and ratio of second-to-first normal stress differences (following linear chains). At large ring fraction, the normal stress ratio of blends seems to follow that of pure rings.

The presented data should be useful to develop a deeper understanding of the intriguing nonlinear response of ring polymers and their blends. In addition, we hope that it will trigger further studies as more experimental and simulation data are clearly needed. Importantly, in addition to simulations and further development and extension of the shear slit model (to include tumbling and model start-up in flow), in-situ or ex-situ structural investigations of the sheared and elongated (e.g. Ref.[8]) rings and ring-linear blends will shed light into the mechanisms of shear deformation and possible tumbling. In this direction, polystyrenes offer the advantage for easy ex-situ studies once quenched to room temperature at different rates (for example, this was done in Ref.[8]). This the subject of current and future investigations.

\section{SUPPLEMENTARY MATERIAL}

Sample characterization: SEC and liquid chromatography at the critical condition.

Nonlinear shear rheology: shear stress growth functions, steady-shear viscosities and normal forces. Nonlinear uniaxial extensional rheology.

\section{Acknowledgements}

Financial support by the Greek General Secretarial for Research \& Technology (ARISTEIA-RINGS), FORTH (Synergy-2019) and the Aage og Johanne Louis-Hansen foundation is gratefully acknowledged. M. R. acknowledges financial support from National Science Foundation under Grant EFMA-1830957 and the National Institutes of Health under Grant P01-HL108808. 


\section{References}

[1] Kapnistos, M., Lang, M., Vlassopoulos, D., Pyckhout-Hintzen, W., Richter, D., Cho, D., Chang, T., and M. Rubinstein, Unexpected power-law stress relaxation of entangled ring polymers. Nature Materials 2008;7:997.

[2] Halverson, J. D., Lee, W. B., Grest, G. S., Grosberg, A. Y., and K. Kremer, Molecular dynamics simulation study of nonconcatenated ring polymers in a melt. I. Statics. The Journal of Chemical Physics 2011;134:204904.

[3] Yan, Z-C, Costanzo, S., Jeong, Y., Chang, T., and D. Vlassopoulos, Linear and nonlinear shear rheology of a marginally entangled ring polymer. Macromolecules 2016;49:1444-1453.

[4] Parisi, D., Ahn, J., Chang, T., Vlassopoulos, D., and M. Rubinstein, Stress relaxation in symmetric ring-linear polymer blends at low ring fractions. Macromolecules 2020;53:16851693.

[5] McKenna, G.B., and D. J. Plazek,. The viscosity of blends of linear and cyclic molecules of similar molecular mass. Polymer Communications 1986;27:304-306.

[6] Roovers, J. Viscoelastic properties of polybutadiene rings. Macromolecules 1988;21:1517-1521.

[7] Peddireddy, K. R., Lee, M., Schroeder, C. M., and R. M. Robertson-Anderson, Viscoelastic properties of ring-linear DNA blends exhibit nonmonotonic dependence on blend composition. Physical Review Research 2020;2:023213.

[8] Borger, A., Wang, W., O’Connor, T. C., Ge, T., Grest, G. S., Jensen, G. V., Ahn, J., Chang, T., Hassager, O., Mortensen, K, Vlassopoulos D., and Q. Huang, Threading-unthreading transition of linear-ring polymer blends in extensional flow. ACS Macro Letters 2020;9:1452-1457.

[9] Huang, Q., Ahn, J., Parisi, D., Chang, T., Hassager, O., Panyukov, S., Rubinstein, M., and D. Vlassopoulos, Unexpected stretching of entangled ring macromolecules. Physical Review Letters 2019;122:208001.

[10] Zhou, Y., Hsiao, K-W, Regan, K. E., Kong, D., McKenna, G. B., and R. M. RobertsonAnderson, and C. S. Schroeder, Effect of molecular architecture on ring polymer dynamics in semidilute linear polymer solutions. Nature Communications 2019;10:1753.

[11] Parisi, D., Costanzo, S., Jeong, Y., Ahn, J., Chang, T., Vlassopoulos, D., Halverson, J. D, Kremer, K., Ge, T., Rubinstein, M., Grest, G. S., Srinin, W., and A. Y. Grosberg, Nonlinear shear rheology of entangled polymer rings Macromolecules 2021; 54: 2811-2827.

[12] Costanzo, S., Ianniruberto, G., Marrucci, G., and D. Vlassopoulos, Measuring and assessing first and second normal stress differences of polymeric fluids with a modular cone-partitioned plate geometry. Rheologica Acta 2018;57:363-376. 
[13] Tsolou, G., Stratikis, N., Baig, C., Stephanou, P. S., and V. G. Mavrantzas, Melt structure and dynamics of unentangled polyethylene rings: Rouse theory, atomistic molecular dynamics simulation, and comparison with the linear analogues. Macromolecules 2010;43:10692-10713.

[14] Tsalikis, D. G., V. G. Mavrantzas, and D. Vlassopoulos, Analysis of Slow Modes in Ring Polymers: Threading of Rings Controls Long-Time Relaxation. ACS Macro Letters 2016;5:755760.

[15] Tsalikis, D. G., Alatas, P. V., Peristeras, L. D., and V. G. Mavrantzas, Scaling laws for the conformation and viscosity of ring polymers in the crossover region around $\mathrm{Me}$ from detailed molecular dynamics simulations. ACS Macro Letters 2018;7:916-920.

[16] Tsamopoulos, A. J., Katsarou, A. F., Tsalikis, D. G., and V. G. Mavrantzas, Shear rheology of unentangled and marginally entangled ring polymer melts from large-scale nonequilibrium molecular dynamics simulations. Polymers 2019;11:1194.

[17] O'Connor, T. C., Ge, T., Rubinstein, M., and G. S., Grest, Topological linking drives anomalous thickening of ring polymers in weak extensional flows. Physical Review Letters 2020;124:027801.

[18] Jeong. Y., Jin, Y., Chang, T., Uhlik, F., and J. Roovers, Intrinsic Viscosity of Cyclic Polystyrene. Macromolecules 2017;50:7770-7776.

[19] Ziebarth, J. D., Gardiner, A. A., Wang, Y., Jeong, Y., Ahn, J., Jin, Y., and T. Chang, Comparison of critical adsorption points of ring polymers with linear polymers. Macromolecules 2016;49:8780-8788.

[20] Lee, H. C., Lee, H., Lee, W., Chang, T., and J. Roovers, Fractionation of cyclic polystyrene from linear precursor by HPLC at the chromatographic critical condition. Macromolecules 2000;33:8119-8121.

[21] Cho, D., Park, S., Kwon, K., Chang, T. Structural Characterization of ring polystyrene by liquid chromatography at the critical condition and MALDI-TOF mass spectrometry. Macromolecules 2001;34:7570-7572.

[22] Tead, S. F., Kramer, E. J., Hadziioannou, G., Antonietti, M., Sillescu, H., Lutz, P., and C. Strazielle, Polymer topology and diffusion: a comparison of diffusion in linear and cyclic macromolecules. Macromolecules 1992;25:3942-7.

[23] McKenna, G. B., Hadziioannou, G., Lutz, P., Hild, G., Strazielle, C., Straupe, C., Rempp, P., and A. J. Kovacs, Dilute solution characterization of cyclic polystyrene molecules and their zeroshear viscosity in the melt. Macromolecules 1987;20:498-512.

[24] McKenna, G. B., Hostetter, B. J., Hadjichristidis, N., Fetters L. J., and D. J. Plazek, A study of the linear viscoelastic properties of cyclic polystyrenes using creep and recovery measurements. Macromolecules 1989;22:1834-1852. 
[25] Rubinstein, M., and R. H. Colby, Polymer Physics. Oxford University Press New York; 2003.

[26] Doi, M., and S. F. Edwards, The theory of polymer dynamics. Oxford Clarendon Press; 1988.

[27] Fetters, L. J., Lohse, D. J., and R. H. Colby, Chain dimensions and entanglement spacings. In: Mark, J. E., Physical Properties of Polymers Handbook, NY Springer New York; 2007, p. 44754.

[28] Doi, Y., Matsubara, K., Ohta, Y., Nakano, T., Kawaguchi, D., Takahashi, Y., Takano, A., and Y. Matsuhita. Melt rheology of ring polystyrenes with ultrahigh purity. Macromolecules 2015;48:3140-3147.

[29] Watanabe, H., Inoue, T., and Y. Matsumiya, Transient conformational change of bead- spring ring chain during creep process. Macromolecules 2006;39:5419-5426.

[30] Wiest, J. M., Burdette, S. R., Liu, T. W., and R. B. Bird, Effect of ring closure on rheological behavior. Journal of Non-Newtonian Fluid Mechanics 1987;24:279-295.

[31] Meissner, J., Garbella, R. W., and J. Hostettler, Measuring normal stress differences in polymer melt shear flow. Journal of Rheology 1989;33:843-864.

[32] Schweizer, T. Measurement of the first and second normal stress differences in a polystyrene melt with a cone and partitioned plate tool. Rheologica Acta 2002;41:337-344.

[33] Walsh, D., and P. Zoller, Standard pressure volume temperature data for polymers. CRC Press; 1995.

[34] Costanzo, S., Huang, Q., Ianniruberto, G., Marrucci, G., Hassager, O., and D. Vlassopoulos, Shear and extensional rheology of polystyrene melts and solutions with the same number of entanglements. Macromolecules 2016;49:3925-3935.

[35] Schweizer, T., and A. Bardow, The role of instrument compliance in normal force measurements of polymer melts. Rheologica Acta 2006;45:393-402.

[36] Bach, A., Rasmussen H. K, and O. Hassager, Extensional viscosity for polymer melts measured in the filament stretching rheometer. Journal of Rheology 2003;47:429-441.

[37] Rasmussen, H. K., Bejenariu, A. G., Hassager, O., and D. Auhl, Experimental evaluation of the pure configurational stress assumption in the flow dynamics of entangled polymer melts. Journal of Rheology 2010;54:1325-1336.

[38] Kolte, M. I., Rasmussen, H. K., and O. Hassager, Transient filament stretching rheometer. Rheologica Acta 1997;36:285-302.

[39] Marín, J. M. R., Huusom, J. K., Alvarez, N. J., Huang, Q., Rasmussen, H. K., Bach, A., Skov, A. L., and O. Hassager, A control scheme for filament stretching rheometers with application to polymer melts. Journal of Non-Newtonian Fluid Mechanics 2013;194:14-22. 
[40] Hou, J-X. Stress relaxation of entangled ring polymer chains in a linear matrix. Journal of Rheology 2020;64:1315-1324.

[41] Doi, Y., Matsumoto, A., Inoue, T., Iwamoto, T., Takano, A., Matsushita, Y., Takahashi, Y., Watanabe, $\mathrm{H}$. Re-examination of terminal relaxation behavior of high-molecular-weight ring polystyrene melts, Rheol. Acta 2017, 56:567-581.

[42] Pasquino, R., Vasilakopoulos, T.C., Jeong, Y.C., Lee, H., Rogers, S., Sakellariou, G., Allgaier, J., Takano, A., Brás, A.R., Chang, T., Gooßen, S., Pyckhout-Hintzen, W., Wischnewski, A., Hadjichristidis, N., Richter, D., Rubinstein, M., Vlassopoulos, D. Viscosity of ring polymer melts. ACS Macro Lett. 2013, 2: 874-878.

[43] Kapnistos, M., Linear and nonlinear rheology of architecturally complex polymers. Ph.D. thesis, University of California, Santa Barbara, 2006

[44] Ge, T., Panyukov, S., and M. Rubinstein, Self-similar conformations and dynamics in entangled melts and solutions of nonconcatenated ring polymers. Macromolecules 2016;49:708722.

[45] Cox, W. P., and E. H. Merz, Correlation of dynamic and steady flow viscosities. Journal of Polymer Science 1958;28:619-622.

[46] Stephanou, P.S., Schweizer, T. Kroger, M.. Communication: Appearance of undershoots in start-up shear Experimental findings captured by tumbling-snake dynamics. J. Chem. Phys. 2017, 146:161101.

[47] Masubuchi, Y., D. Vlassopoulos, D., G. Ianniruberto, G., G. Marrucci, G. Wall slip in primitive chain network simulations of shear startup of entangled polymers and its effect on the shear stress undershoot. J. Rheol., 2021, 65, 213-223.

[48] Li, Y., Hsiao, K-W, Brockman, C. A., Yates, D. Y., Robertson-Anderson, R. M., Kornfield, J. A., San Francisco, M. J., Schroeder, C. M., and G. B. McKenna, When ends meet: Circular DNA stretches differently in elongational flows. Macromolecules 2015;48:5997-6001.

[49] Stephanou, P. S., and M. Kröger, Non-constant link tension coefficient in the tumbling-snake model subjected to simple shear. The Journal of Chemical Physics 2017;147:174903.

[50] Hsiao, K-W, Schroeder, C. M., and C. E. Sing, Ring polymer dynamics are governed by a coupling between architecture and hydrodynamic interactions. Macromolecules 2016;49:19611971.

[51] Liebetreu M, Ripoll M, Likos CN. Trefoil knot hydrodynamic delocalization on sheared ring polymers. ACS Macro Letters 2018;7:447-452. 
[52] Liebetreu, M., and C. N. Likos, Hydrodynamic inflation of ring polymers under shear. Communications Materials 2020;1:1-11.

[53] Chen, W., Chen, J., and L. An, Tumbling and tank-treading dynamics of individual ring polymers in shear flow. Soft Matter 2013;9:4312-4318.

[54] Carreau, P. J. Rheological equations from molecular network theories. J. Rheol. 1972, 16, 99-127.

[55] Yasuda, K. Investigation of the analogies between viscometric and linear viscoelastic properties of polystyrene fluids (579 pages). Ph.D. thesis, MIT, 1979; URL: https://dspace.mit.edu/handle/1721.1/ 16043.

[56] Yasuda, K.; Armstrong, R. C.; Cohen, R. E. Shear flow properties of concentrated solutions of linear and star branched polystyrenes. Rheol. Acta 1981, 20, 163-178.

[57] Wang, S-Q. Nonlinear polymer rheology. Wiley Online Library; 2018.

[58] Graham, R. S., Likhtman, A. E., McLeish, T. C., and S. T. Milner. Microscopic theory of linear, entangled polymer chains under rapid deformation including chain stretch and convective constraint release. Journal of Rheology 2003;47:1171-1200.

[59] Auhl, D., Ramirez, J., Likhtman, A. E., Chambon, P., and C. Fernyhough, Linear and nonlinear shear flow behavior of monodisperse polyisoprene melts with a large range of molecular weights. Journal of Rheology 2008;52:801-835.

[60] Xie, S-J, and K. S. Schweizer, Consequences of delayed chain retraction on the rheology and stretch dynamics of entangled polymer liquids under continuous nonlinear shear deformation. Macromolecules 2018;51:4185-4200.

[61] Schweizer, K. S., and S-J Xie, Physics of the stress overshoot and chain stretch dynamics of entangled polymer liquids under continuous startup nonlinear shear. ACS Macro Letters 2018;7:218-222.

[62] Yoon, J., Kim, J., and C. Baig, Nonequilibrium molecular dynamics study of ring polymer melts under shear and elongation flows: A comparison with their linear analogs. Journal of Rheology 2016;60:673-685.

[63] Xu, X., Chen, J., and L. An, Shear thinning behavior of linear polymer melts under shear flow via nonequilibrium molecular dynamics. The Journal of Chemical Physics 2014;140:174902.

[64] Jeong, S., Cho, S., Kim, J. M., and C. Baig, Interfacial molecular structure and dynamics of confined ring polymer melts under shear flow. Macromolecules 2018;51:4670-4677.

[65] Nielsen, J. K., Rasmussen, H. K., Hassager, O., and G. H. McKinley, Elongational viscosity of monodisperse and bidisperse polystyrene melts. Journal of Rheology 2006;50:453-476. 
[66] Marrucci, G., and G. Ianniruberto, Interchain pressure effect in extensional flows of entangled polymer melts. Macromolecules 2004;37:3934-3942.

[67] Laun, H. M., Prediction of elastic strains of polymer melts in shear and elongation. Journal of Rheology 1986;30:459-501.

[68] Sharma, V., and G. H. McKinley, An intriguing empirical rule for computing the first normal stress difference from steady shear viscosity data for concentrated polymer solutions and melts. Rheologica Acta 2012;51:487-95. 


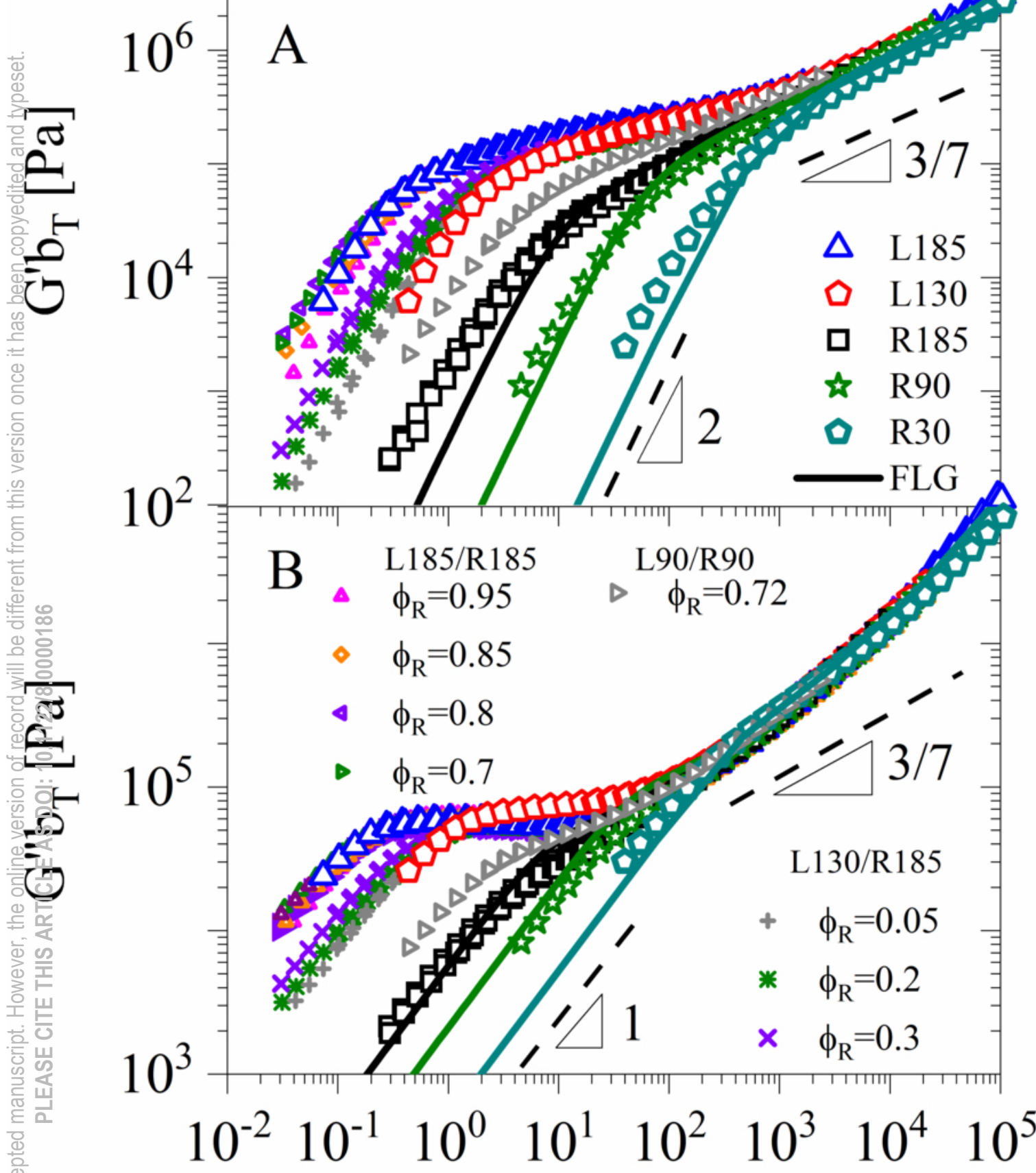
$\omega \mathrm{a}_{\mathrm{T}}[\mathrm{rad} / \mathrm{s}]$ 


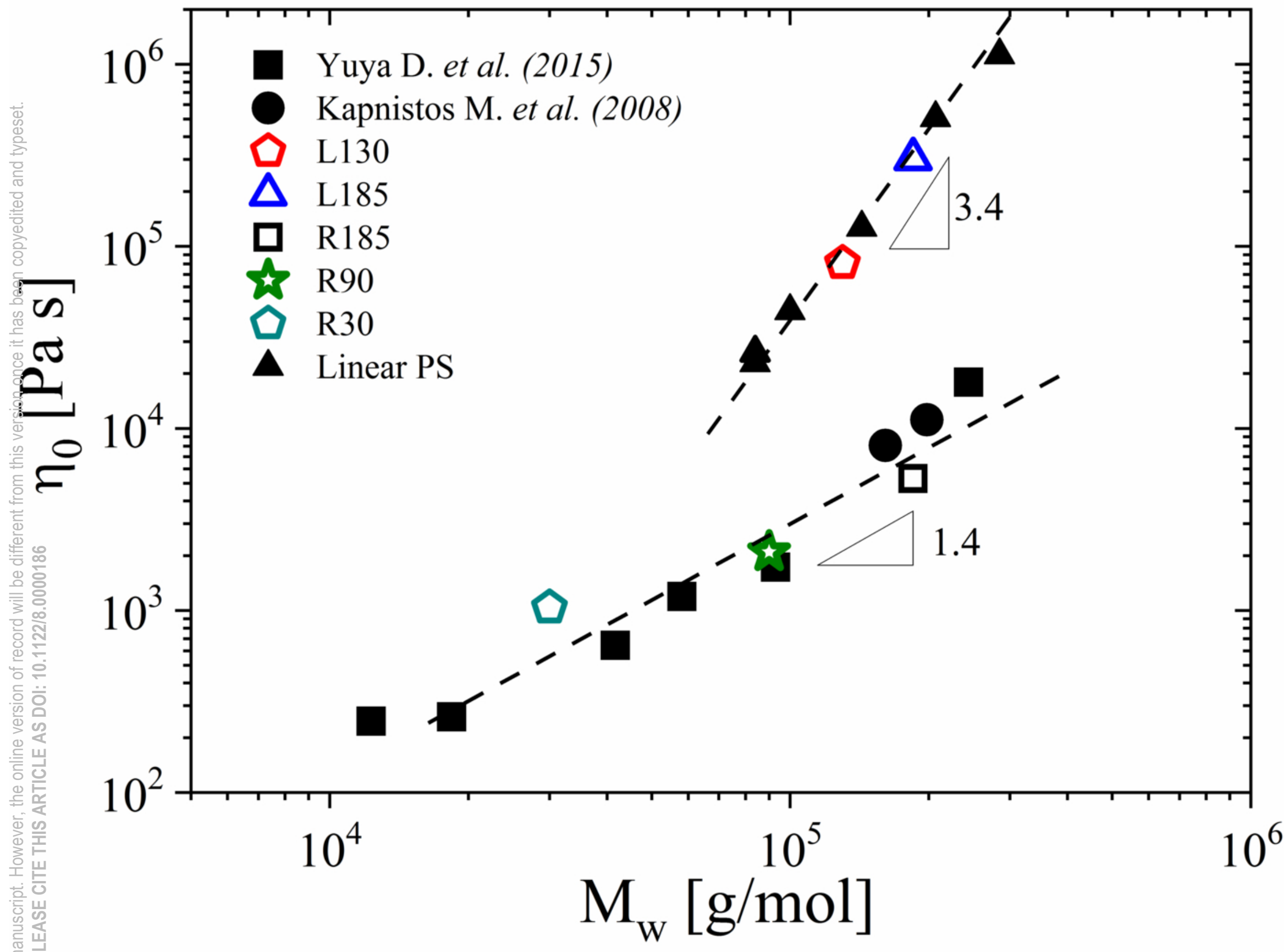



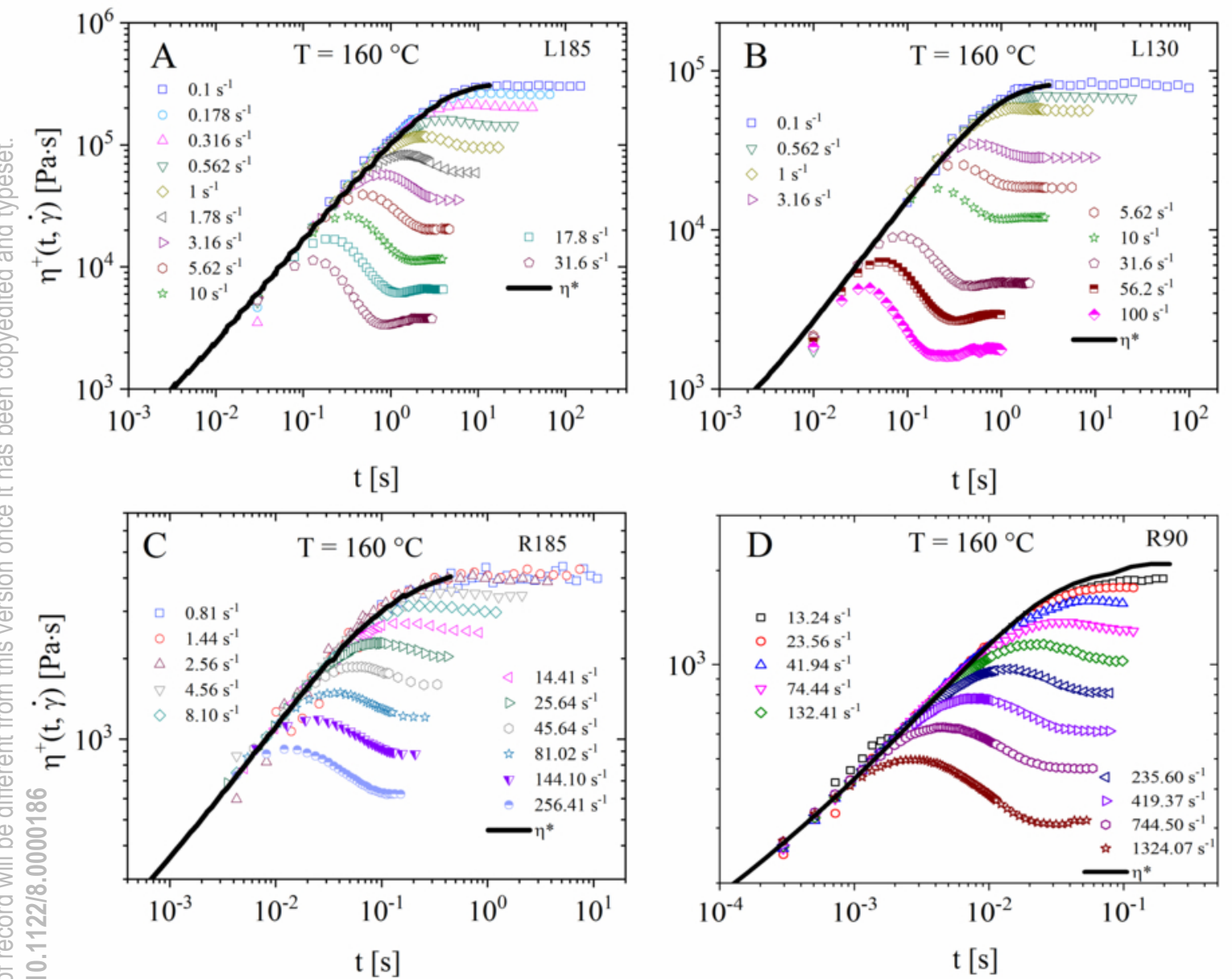

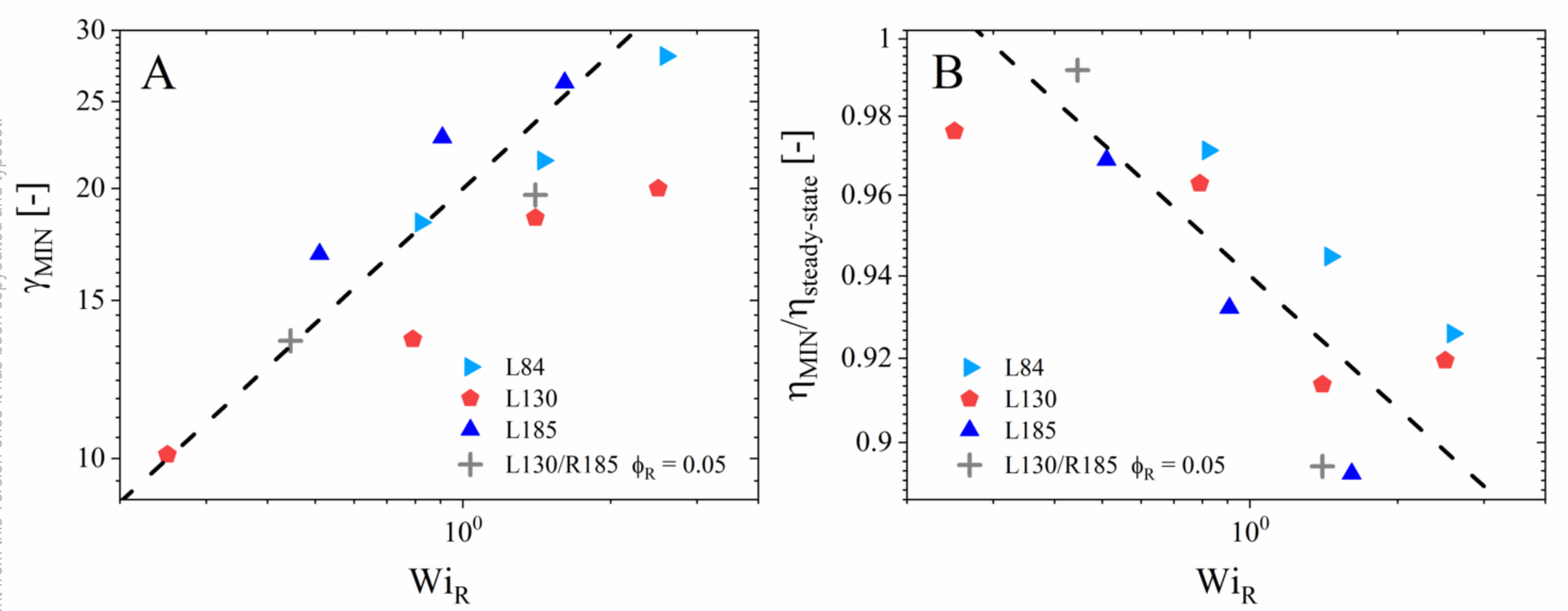


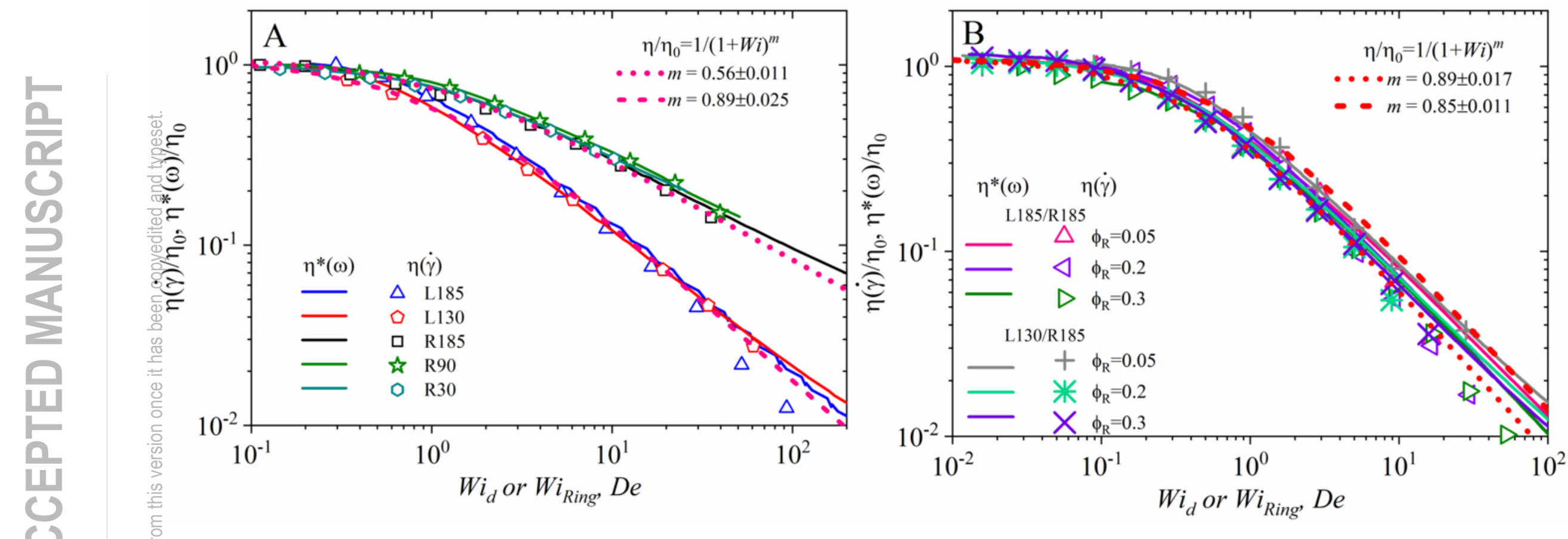




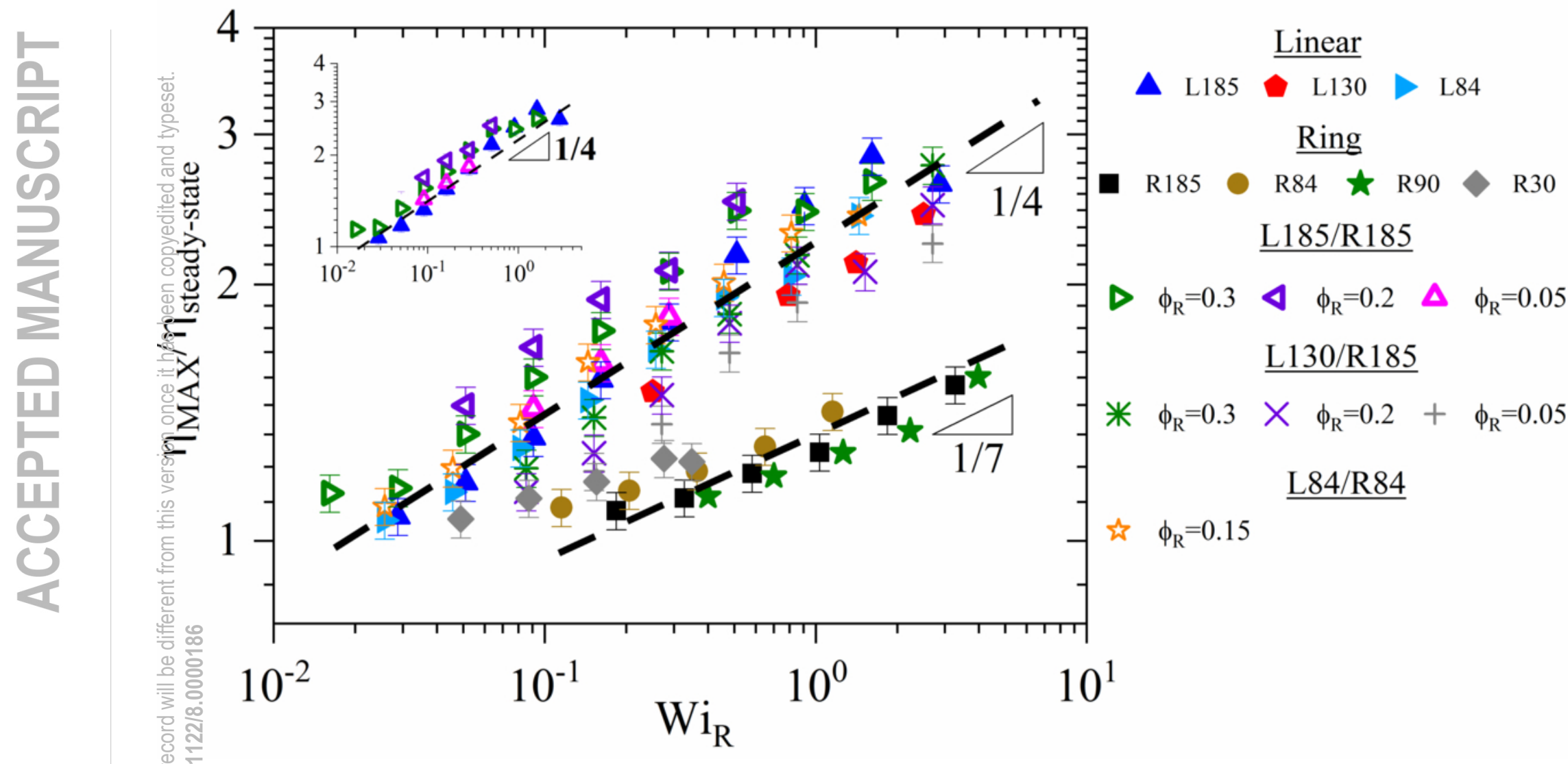




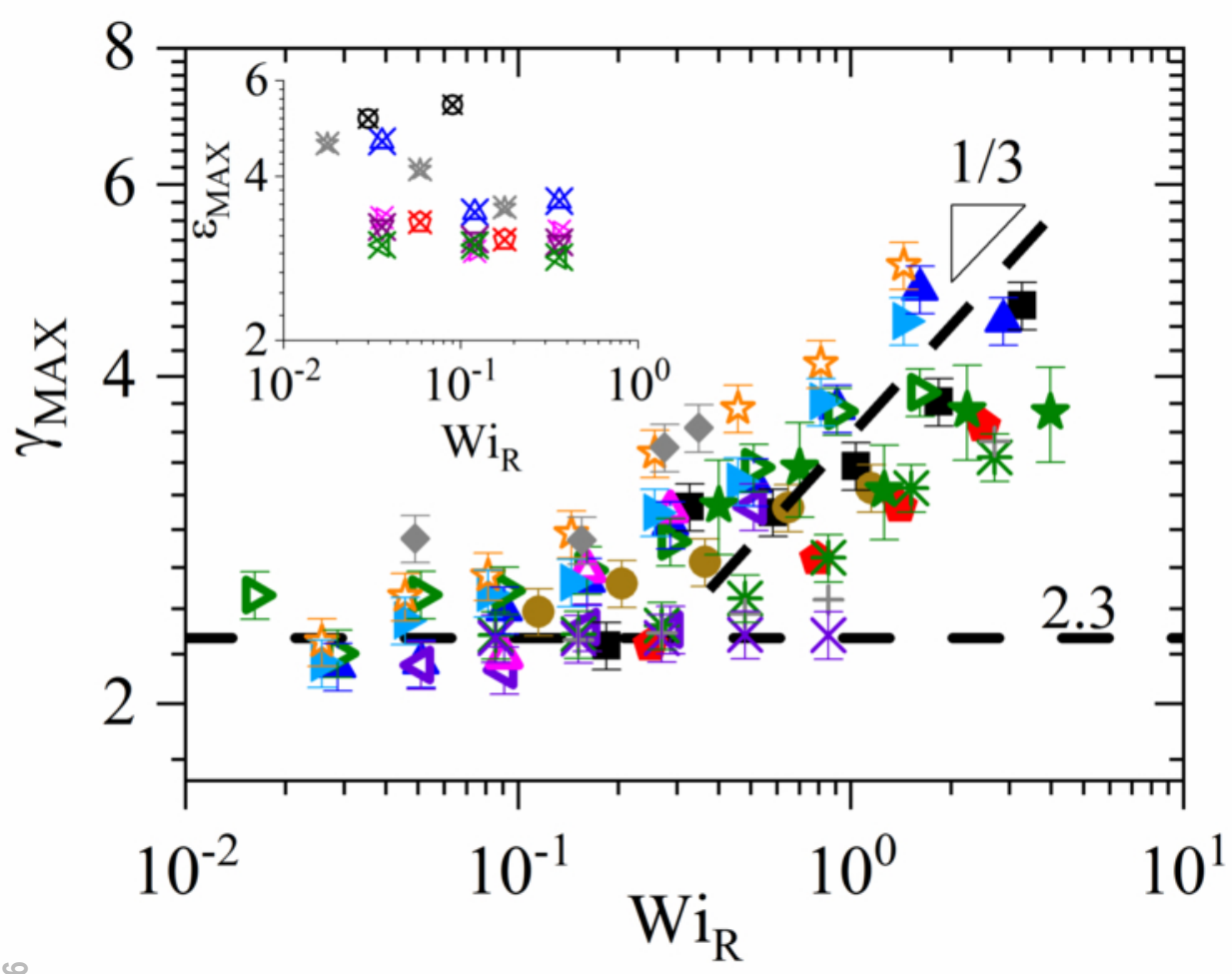

Shear: $\gamma_{\text {MAX }}$

$\Delta$ L185 $\frac{\text { Linear }}{\text { L130 }}>$ L84
R185 $\star \frac{\text { Ring }}{\text { R90 }}{ }_{\text {R84 }} \diamond \mathrm{R} 30$ L185/R185

$\triangleright \phi_{\mathrm{R}}=0.3 \triangleleft \phi_{\mathrm{R}}=0.2 \Delta \phi_{\mathrm{R}}=0.05$ L130/R185

米 $\phi_{\mathrm{R}}=0.3 \times \phi_{\mathrm{R}}=0.2+\phi_{\mathrm{R}}=0.05$

is $\phi_{\mathrm{R}}=0.15 \stackrel{\mathrm{L} 84 / \mathrm{R} 84}{ }$

Extension: $\varepsilon_{\mathrm{MAX}}$

L185 凶 $\underset{\text { Ling }}{\frac{\text { Linear }}{\text { Ring }}}$

邓 R185

L185/R185

\ $\phi_{\mathrm{R}}=0.3$ 邓 $\phi_{\mathrm{R}}=0.2$ 民 $\phi_{\mathrm{R}}=0.05$ 谈 $\phi_{\mathrm{R}}=0.2 \underline{\mathrm{L} 130 / \mathrm{R} 185}$ 


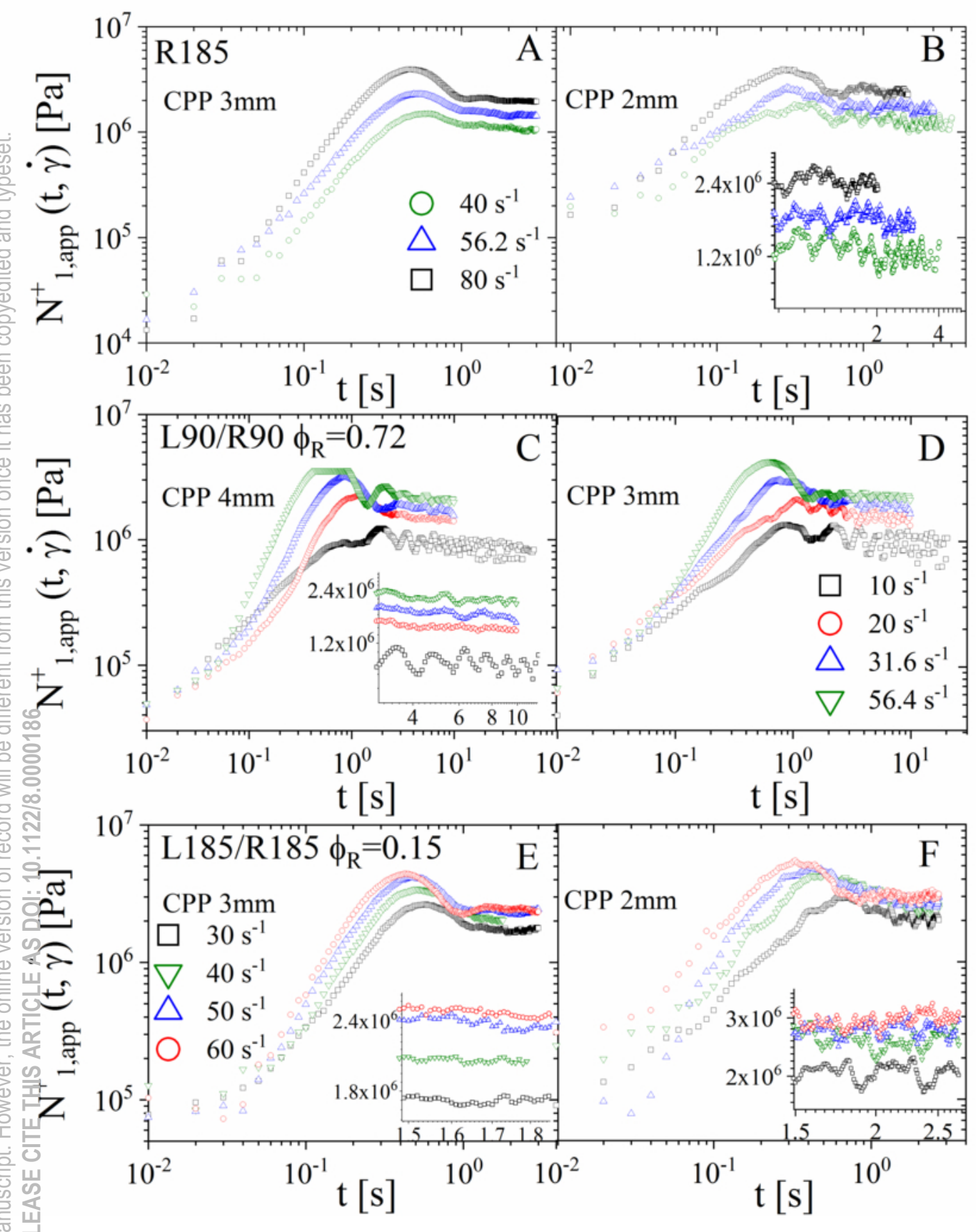




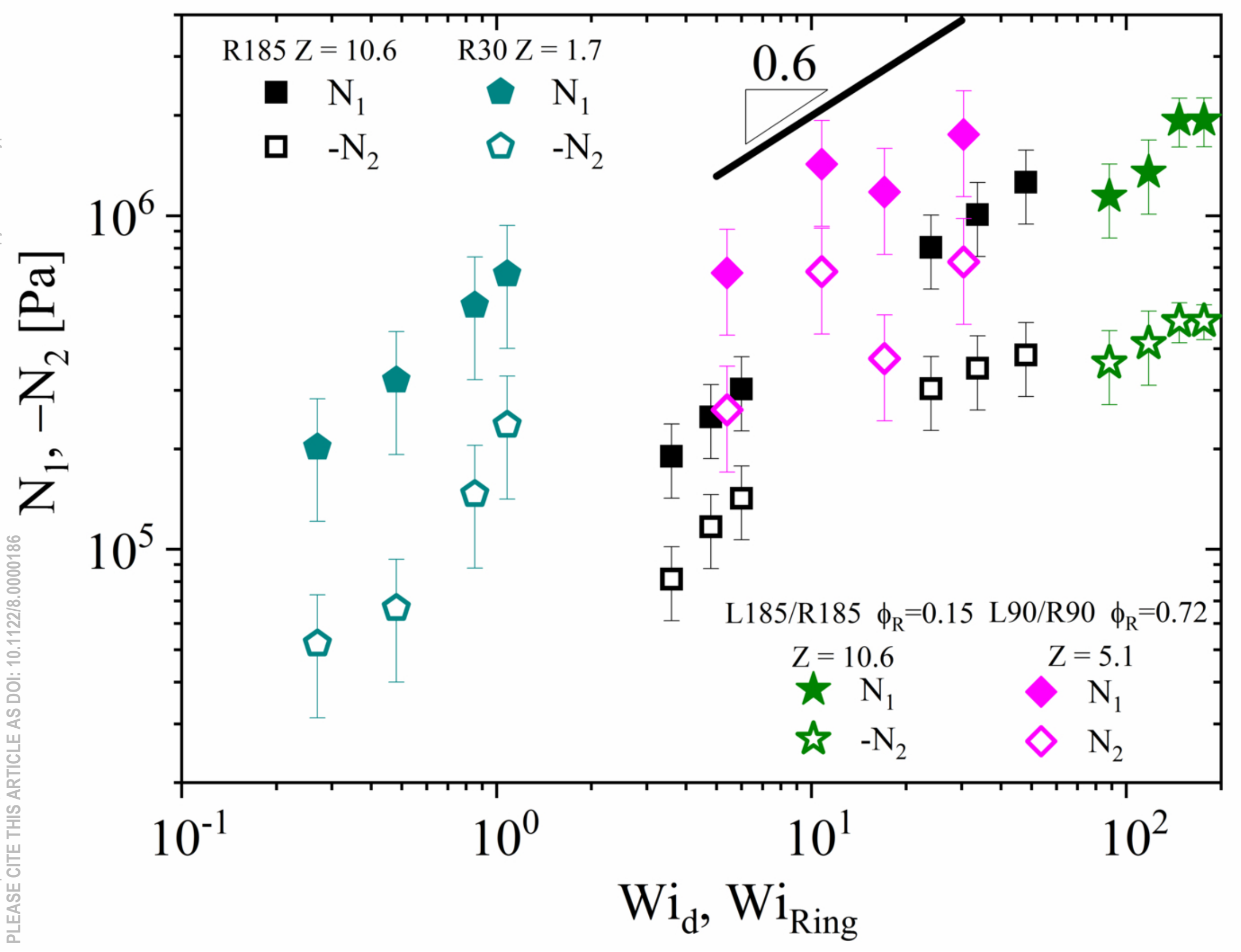




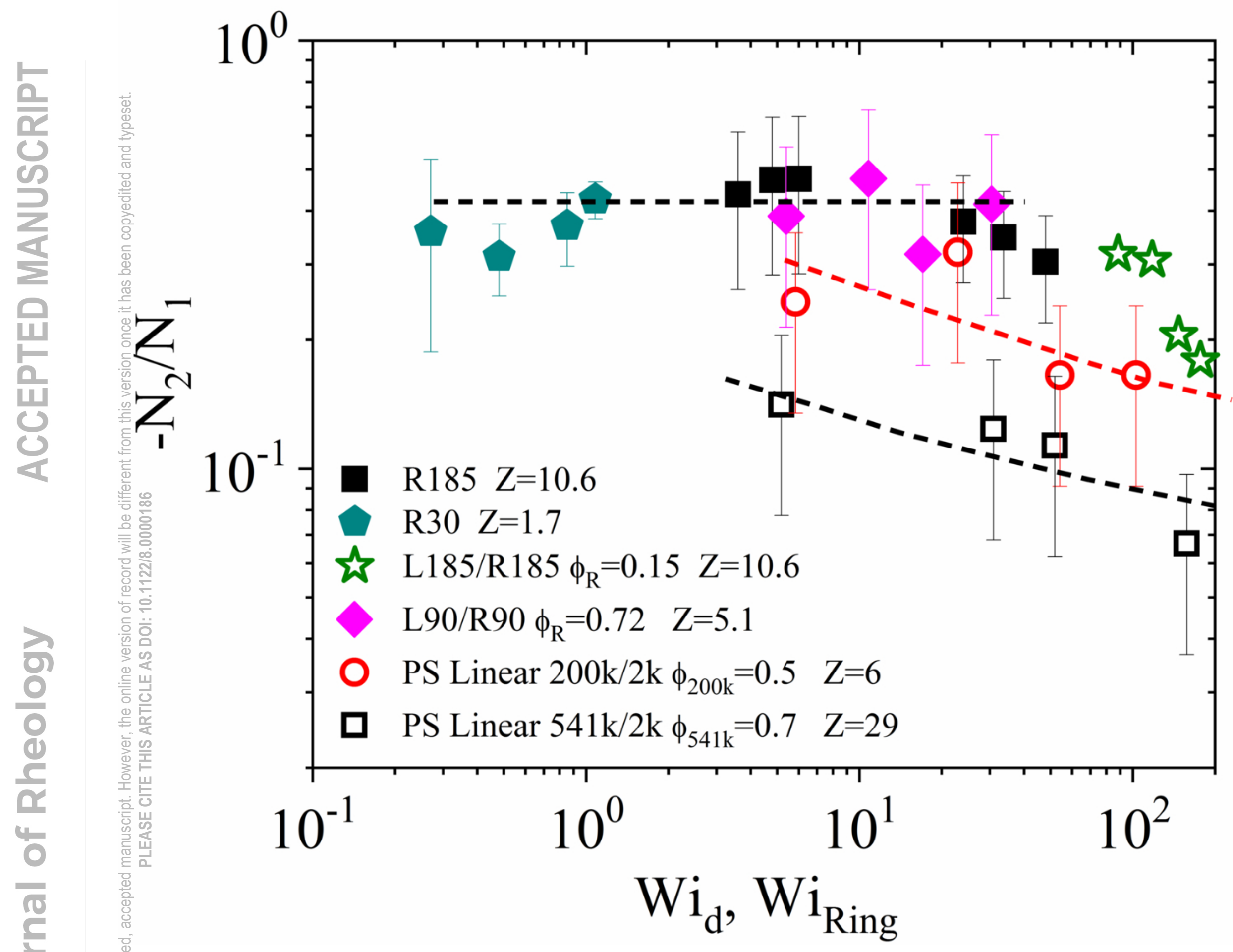




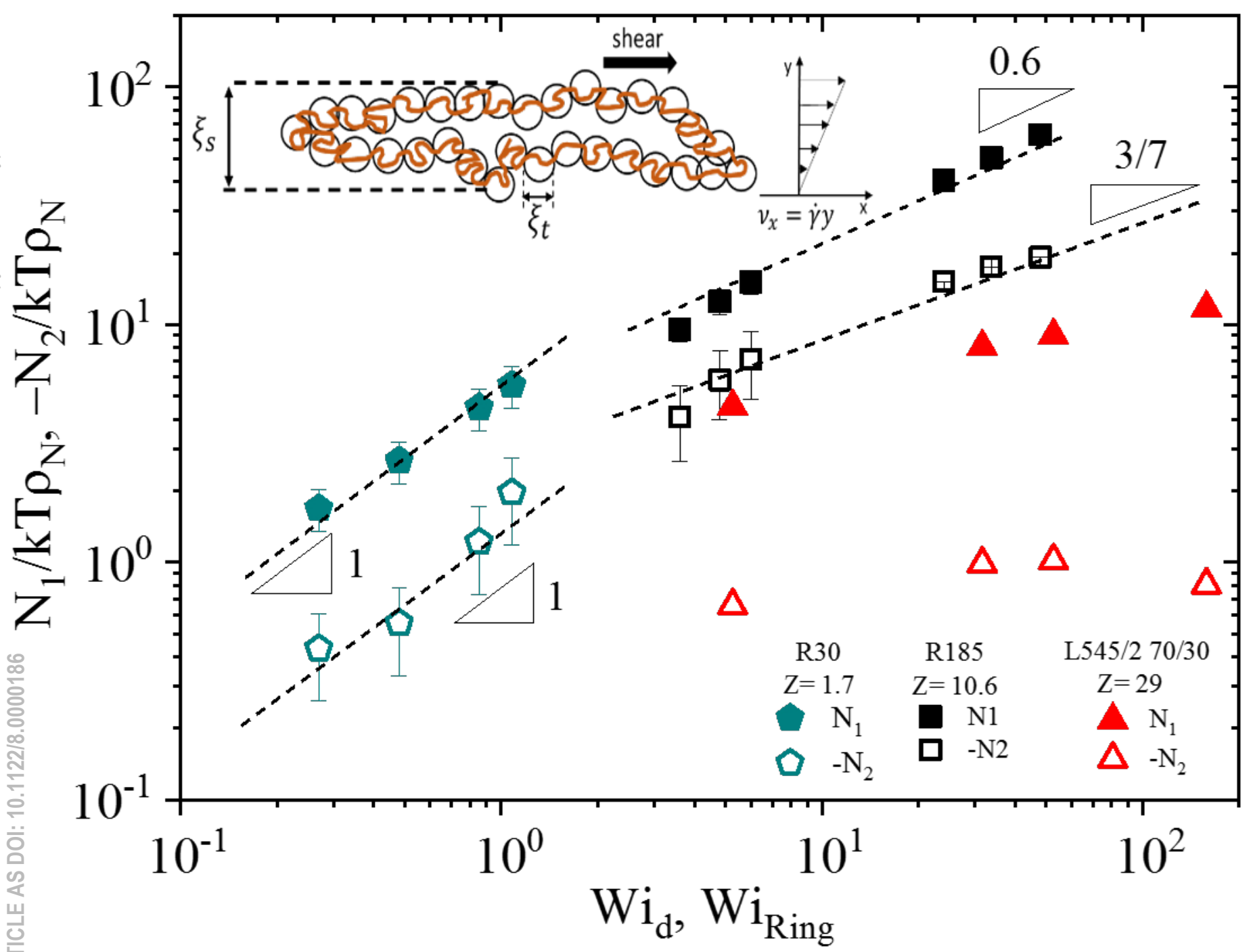




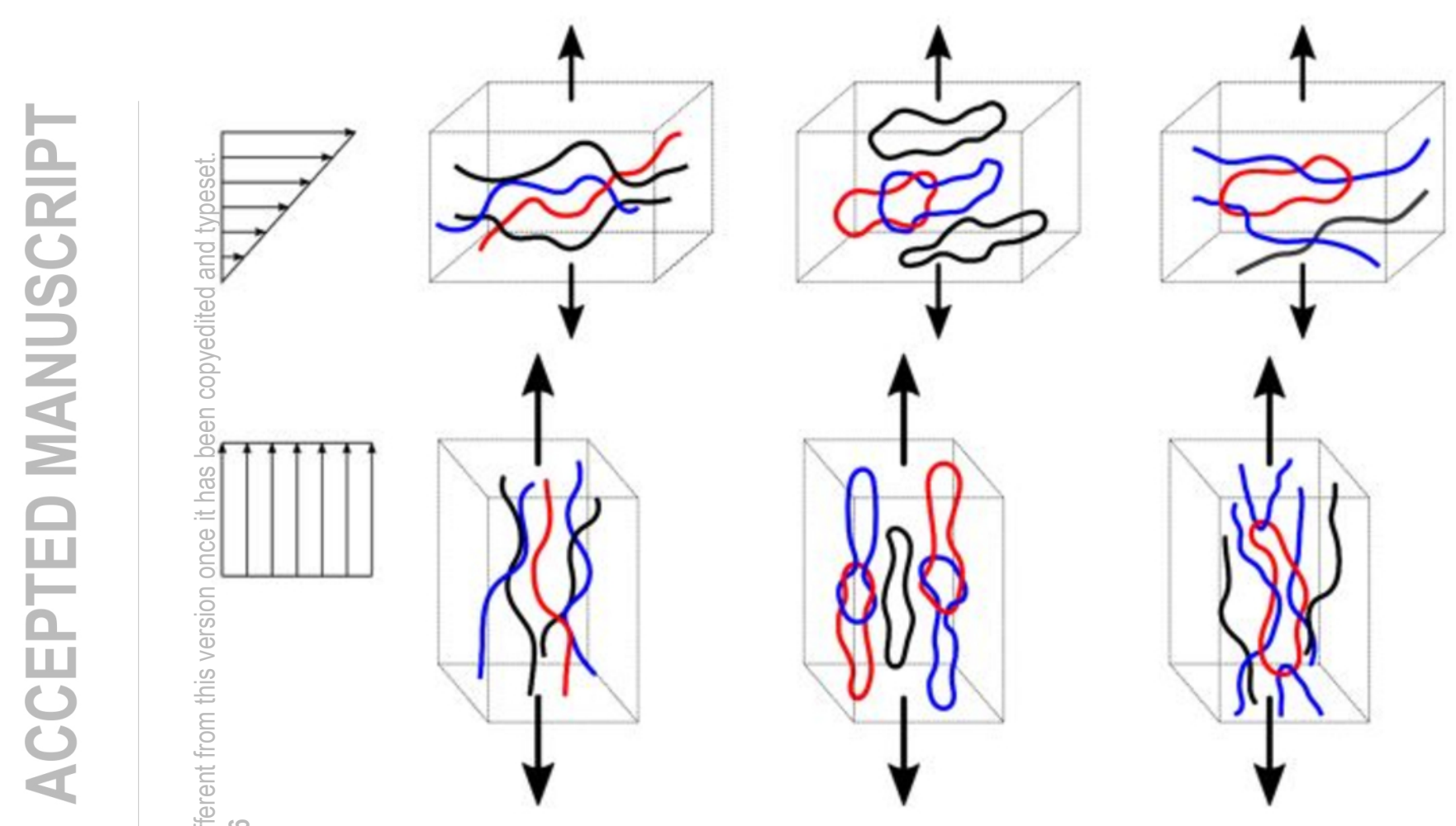




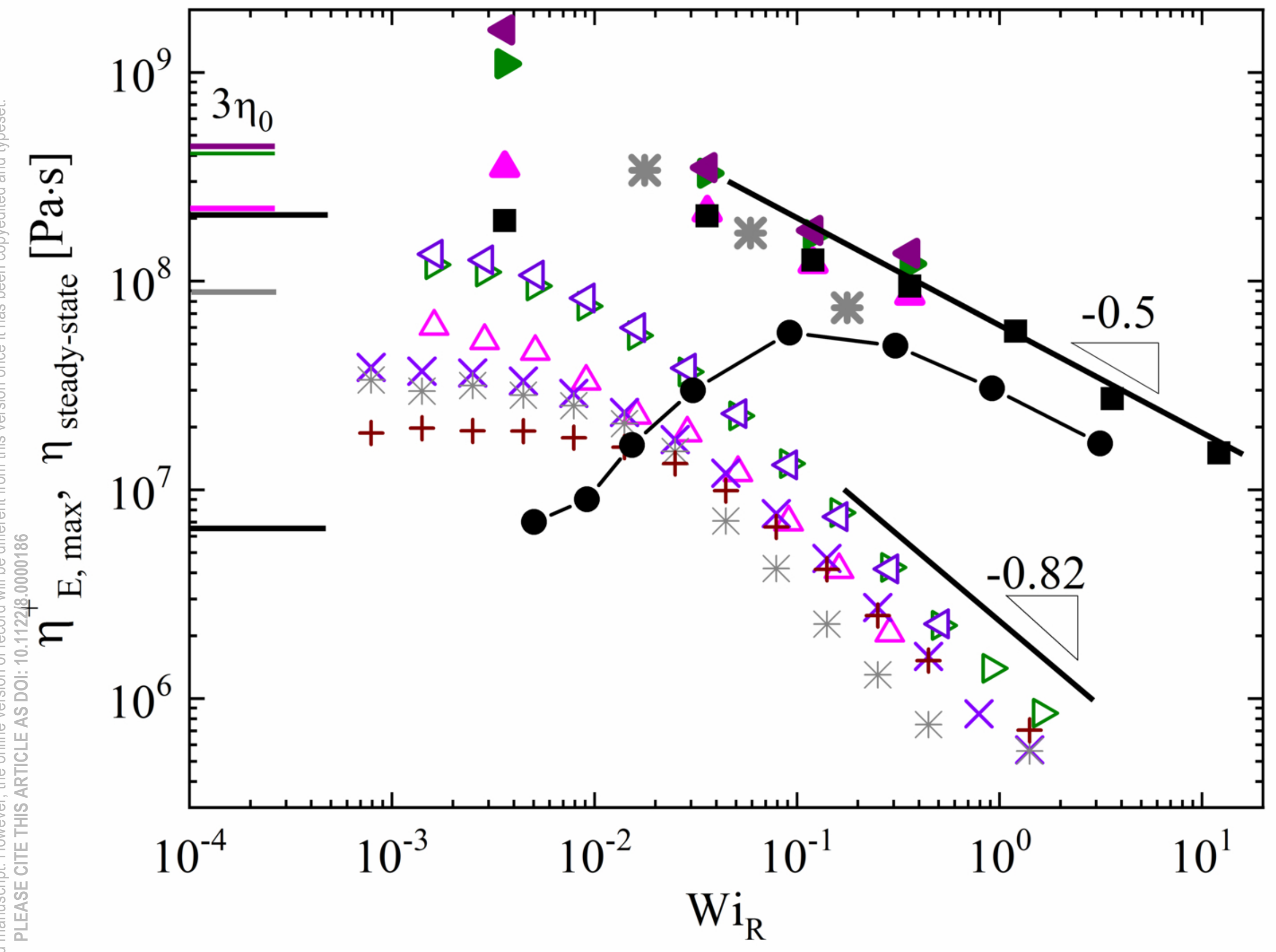

\section{Extension}

- L185

-O-R185

Extension

Shear

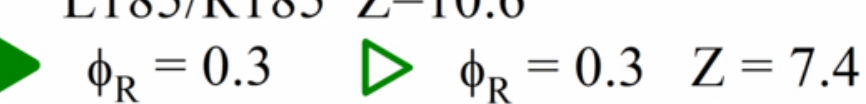

$\phi_{\mathrm{R}}=0.2 \quad \triangleleft \quad \phi_{\mathrm{R}}=0.2 \quad \mathrm{Z}=8.5$

$\triangle \phi_{\mathrm{R}}=0.05 \triangle \phi_{\mathrm{R}}=0.05 \mathrm{Z}=10$ L130/R185 Z=7.4

$+\phi_{\mathrm{R}}=0.05 \mathrm{Z}=7.0$

$\varkappa^{\prime} \phi_{R}=0.2$

$$
\begin{array}{ll}
\text { * } \phi_{\mathrm{R}}=0.2 & \mathrm{Z}=6.0 \\
\times \quad \phi_{\mathrm{R}}=0.3 & \mathrm{Z}=5.2
\end{array}
$$


